\title{
SOLVING THE OCTIC BY ITERATION IN SIX DIMENSIONS
}

\author{
SCOTT CRASS
}

\begin{abstract}
The requirement for solving a polynomial is a means of breaking its symmetry, which in the case of the octic, is that of the symmetric group $\mathcal{S}_{8}$. Its eight-dimensional linear permutation representation restricts to a six-dimensional projective action. A mapping of complex projective 6 -space with this $\mathcal{S}_{8}$ symmetry can provide the requisite symmetrybreaking tool.

The article describes some of the $\mathcal{S}_{8}$ geometry in $\mathbf{C P}^{6}$ as well as a special $\mathcal{S}_{8}$-symmetric rational map in degree four. Several basins-of-attraction plots illustrate the map's geometric and dynamical properties. The work culminates with an explicit algorithm that uses this map to solve a general octic. A concluding discussion treats the generality of this approach to equations in higher degree.
\end{abstract}

\section{Overview}

In Crass 2001, I develop a solution to the quintic that relies on a single iteration in three dimensions. Given almost any quintic $p$, there is a map on complex projective 3 -space $\mathbf{C P}^{3}$ whose dynamics provides for a root of $p$. This approach is geometric: the map has the $\mathcal{S}_{5}$ symmetry of the general quintic.

The present paper extends this work to the eighth-degree equation. At its core is an $\mathcal{S}_{8}$-symmetric map on $\mathbf{C P}^{6}$ whose geometric behavior is connected to a special configuration of lines. Motivating this general project is a desire to develop solutions to equations that utilize symmetrical and geometrically elegant dynamical systems. I do not consider the numerical aspects of the algorithm. However, since the map converges very rapidly, numerical considerations might well be of interest. Indeed, the geometry associated with the map accounts for the rapid convergence.

In addition, the work establishes the existence of a method for all degrees greater than four that is analogous to the eighth-degree case. This involves showing that there is an infinite family of maps - one for each dimension greater than two-with special geometric properties.

Finally, these maps add to the examples of complex dynamics in several dimensions. This recently-active and difficult field seems to be in need of examples that are not concocted for purposes of illustration.

The work unfolds in four stages: 1) some background geometry, 2) a special map with $\mathcal{S}_{8}$ symmetry, 3) a solution to the octic based on the preceding stages, 4) a consideration of whether the octic algorithm generalizes to higher degree equations.

Section 2; $\mathcal{S}_{8}$ geometry. The setting here is $\mathbf{C P}^{6}$ upon which the symmetric group $\mathcal{S}_{8}$ acts. Finding a map with special $\mathcal{S}_{8}$ geometry requires some familiarity with this action. We will consider some features associated with the map that emerges in the second stage.

Date: March 4, 2020.

Key words and phrases. complex dynamics, equivariant maps, polynomial equations, symmetric group. 
Indeed, the discovery of this map derives from an awareness of the algebraic and geometric surroundings:

- coordinate systems on $\mathbf{C P}^{6}$

- the structure of certain special orbits of points, lines, planes, and hyperplanes

- the system of $\mathcal{S}_{8}$-invariant polynomials - the building-blocks for maps that are $\mathcal{S}_{8^{-}}$ symmetric.

Section 3: Maps with $\mathcal{S}_{8}$ symmetry. At this stage, we exploit our geometric understanding to discover empirically a map associated with the complete graph on eight vertices - an 8-point $\mathcal{S}_{8}$ orbit. The discussion turns to its geometric and dynamical behaviorempirical testing suggests that the 8-point orbit is the only attractor. However, whether it possesses this or another desired global dynamical property is not known. In light of substantial experimental and graphical evidence, I attribute these properties to the map in conjectures.

Section 4: Dynamical solution to the octic. A special family of octics corresponds to a rigid family $\mathcal{E}$ of $\mathcal{S}_{8}$-symmetric maps on $\mathbf{C P}^{6}$. 'Rigidity' means that each member of $\mathcal{E}$ is conjugate to a single reference map $f$. Thus, associated with an octic $p$ is a map $g_{p}=\phi_{p} f \phi_{p}^{-1}$ that we iterate. Using $\mathcal{S}_{8}$ tools, the dynamical output-conjecturally, a single $\mathcal{S}_{8}$ orbit-provides for an approximate solution to $p=0$. Since almost any octic $p$ transforms into the special family, the solution is general.

Note: Up to this point, the exposition follows that of Crass 2001 which the reader can consult for details.

Section 5: Generalization: Solving the $n$ th-degree equation by iteration in $n-2$ dimensions. The geometric and dynamical description of the octic-solving map has an analogue for each permutation-based $\mathcal{S}_{n}$ action on $\mathbf{C P}^{n-2}$ with $n \geq 5$. Here, we can show that there is always a map with the desired special properties. Given such a map for which the $n$-point orbit is the attractor, the solution algorithm for the octic generalizes to one for the $n$th degree equation.

\section{2. $\mathcal{S}_{8}$ ACTS ON $\mathbf{C P}^{6}$}

The search for a special $\mathcal{S}_{8}$-symmetric map begins with a faithful action of $\mathcal{S}_{8}$. Klein's approach to the $n$th degree equation was to look for the lowest dimensional faithful action of $\mathcal{S}_{n}$ or the alternating group $\mathcal{A}_{n}$. For $n<8$, there are special actions of either $\mathcal{S}_{n}$ or $\mathcal{A}_{n}$; that is, there are faithful representations that do not derive directly from permutations on $\mathbf{C}^{n}$. However, special geometry - at least for linear actions - ends at $n=7$. When $n>7$, the space of least dimension on which $\mathcal{S}_{n}$ or $\mathcal{A}_{n}$ acts faithfully is $\mathbf{C P}^{n-2}$. Wiman 1899]

The permutation action of the symmetric group $\mathcal{S}_{8}$ on $\mathbf{C}^{8}$ preserves the hyperplane

$$
\mathcal{H}_{x}=\left\{\sum_{k=1}^{8} x_{k}=0\right\} \simeq \mathbf{C}^{7}
$$

and, thereby, restricts to a faithful seven-dimensional irreducible representation. This $\mathbf{C}^{7}$ action projects one-to-one to a group $\mathcal{G}_{8 \text { ! }}$ on $\mathbf{C P}^{6}$.

2.1. Coordinates. For many purposes, the most perspicuous geometric description of $\mathcal{G}_{8 \text { ! }}$ employs eight coordinates that sum to zero. One advantage is the simple expression of the $\mathcal{S}_{8}$-duality between points and hyperplanes. In general, for a finite action $\mathcal{G}$ whose matrix 
representatives are unitary, a point $a$ is $\mathcal{G}$-dual to the hyperplane

$$
\mathcal{L}=\{\bar{a} \cdot x=0\} .
$$

Consequently, $a$ and $\mathcal{L}$ have the same stabilizer in $\mathcal{G}$. Since the action of $\mathcal{S}_{8}$ on $\mathbf{C}^{7}$ is orthogonal, a point

$$
a=\left[a_{1}, a_{2}, a_{3}, a_{4}, a_{5}, a_{6}, a_{7}, a_{8}\right]_{\sum a_{k}=0} \in \mathbf{C P}^{6}
$$

corresponds to the hyperplane

$$
\{a \cdot x=0\}=\left\{\sum_{k=1}^{8} a_{k} x_{k}=0\right\} .
$$

(Square brackets indicate homogeneous coordinates.)

A system of seven hyperplane coordinates describes the hyperplane $\mathcal{H}_{u}$. It arises from the "hermitian" change of variable

$$
u=H x \quad x=\overline{H^{T}} u \quad H=\frac{1}{2 \sqrt{2}}\left(\begin{array}{cccccccc}
1 & \omega & i & \omega^{3} & -1 & \omega^{5} & -i & \omega^{7} \\
1 & i & -1 & -i & 1 & i & -1 & -i \\
1 & \omega^{3} & -i & \omega & -1 & \omega^{7} & i & \omega^{5} \\
1 & -1 & 1 & -1 & 1 & -1 & 1 & -1 \\
1 & \omega^{5} & i & \omega^{7} & -1 & \omega & -i & \omega^{3} \\
1 & -i & -1 & i & 1 & -i & -1 & i \\
1 & \omega^{7} & -i & \omega^{5} & -1 & \omega^{3} & i & \omega
\end{array}\right)
$$

where $\omega=e^{\pi i / 4}$ and the choice of scalar factor gives

$$
H \overline{H^{T}}=I_{7} \quad \overline{H^{T}} H=\left(a_{i j}\right) \quad a_{i j}=\left\{\begin{array}{ll}
-\frac{7}{8} & i=j \\
\frac{1}{8} & i \neq j
\end{array} \quad i, j=1, \ldots 8 .\right.
$$

2.2. Invariant polynomials. According to the fundamental result on symmetric functions the $n$ elementary symmetric functions of degrees one through $n$ generate the ring of $\mathcal{S}_{n^{-}}$ invariant polynomials. Since the $\mathcal{S}_{8}$ action on $\mathbf{C P}^{6}$ occurs where the degree- 1 symmetric polynomial vanishes, there are seven generating $\mathcal{G}_{8 !}$-invariants. By Newton's identities, the power sums

$$
F_{k}(x)=\sum_{\ell=1}^{8} x_{\ell}^{k} \quad k=2, \ldots, 8
$$

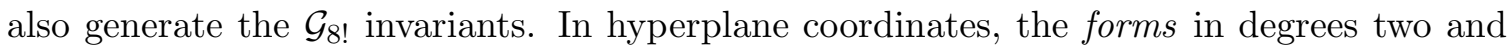
three are

$$
\begin{aligned}
\Phi_{2}(u)= & F_{2}\left(\overline{H^{T}} u\right)=u_{4}^{2}+2 u_{3} u_{5}+2 u_{2} u_{6}+2 u_{1} u_{7} \\
\Phi_{3}(u)= & \frac{3}{2 \sqrt{2}}\left(u_{2} u_{3}^{2}+u_{2}^{2} u_{4}+2 u_{1} u_{3} u_{4}+2 u_{1} u_{2} u_{5}+u_{1}^{2} u_{6}+u_{5}^{2} u_{6}+u_{4} u_{6}^{2}\right. \\
& \left.+2 u_{4} u_{5} u_{7}+2 u_{3} u_{6} u_{7}+u_{2} u_{7}^{2}\right)
\end{aligned}
$$


The remaining five generating invariants $\Phi_{k}(u)$ arise algebraically from these two. Classical techniques show that a relative invariant - invariant up to a multiplicative characterresults from taking the determinant of the bordered hessian

$$
B H(F, G, J)=\left(\begin{array}{cccc} 
& & & \frac{\partial G}{\partial x_{1}} \\
& H(F) & & \vdots \\
& & & \frac{\partial G}{\partial x_{n}} \\
\frac{\partial J}{\partial x_{1}} & \ldots & \frac{\partial J}{\partial x_{n}} & 0
\end{array}\right)
$$

of three $\mathrm{GL}_{n}(\mathbf{C})$ invariants $F, G$, and $J$. The $n \times n$ matrix $H(F)$ is the hessian of $F$.

Proposition 2.1. Given $T \in \mathrm{GL}_{n}(\mathbf{C})$ and $B(x)=\operatorname{det}(B H(F(x), G(x), J(x)))$ for invariants $F, G, J$,

$$
B(T x)=(\operatorname{det} T)^{-2} B(x) .
$$

For the permutation action of $\mathcal{S}_{8}$, this results in an absolute invariant that is expressible in terms of the generators $\Phi_{k}$. The following result will serve a subsequent computational purpose. (Many of this work's results arise from Mathematica computations.)

Proposition 2.2. With

$$
\begin{array}{ll}
G_{4}=\operatorname{det}\left(B H\left(\Phi_{2}, \Phi_{3}, \Phi_{3}\right)\right) & G_{5}=\operatorname{det}\left(B H\left(\Phi_{2}, \Phi_{3}, \Phi_{4}\right)\right) \\
G_{6}=\operatorname{det}\left(B H\left(\Phi_{2}, \Phi_{4}, \Phi_{4}\right)\right) & G_{7}=\operatorname{det}\left(B H\left(\Phi_{2}, \Phi_{4}, \Phi_{5}\right)\right) \\
G_{8}=\operatorname{det}\left(B H\left(\Phi_{2}, \Phi_{5}, \Phi_{5}\right)\right), &
\end{array}
$$

the "power-sum" invariants are given by

$$
\begin{aligned}
\Phi_{4} & =\frac{1}{576}\left(72 \Phi_{2}^{2}+G_{4}\right) & \Phi_{5} & =\frac{1}{768}\left(96 \Phi_{2} \Phi_{3}+G_{5}\right) \\
\Phi_{6} & =\frac{1}{960}\left(120 \Phi_{2} \Phi_{4}+G_{6}\right) & \Phi_{7} & =\frac{1}{1280}\left(160 \Phi_{3} \Phi_{4}+G_{7}\right) \\
\Phi_{8} & =\frac{1}{1600}\left(200 \Phi_{4}^{2}+G_{8}\right) . & &
\end{aligned}
$$

2.3. Special orbits. The 6 -dimensional $\mathcal{S}_{8}$ action comes in both real and complex versions. This means that, in the standard $x$ coordinates, $\mathcal{G}_{8 \text { ! }}$ acts on $\mathcal{R}$ - the $\mathbf{R P}^{6}$ of points with real components. TABLE 2.1 enumerates some special orbits contained in $\mathcal{R}$. For ease of expression, I will refer to special points (or lines, planes, etc.) in terms of the orbit size: "8-points" (28-lines, 56-planes, 28-hyperplanes). Also, these points receive a symbolic description in reference to orbit size (superscript) and coordinate expression (subscript).

Corresponding to each special point $a$ is the hyperplane $\{a \cdot x=0\}$. In the case of the 28-points

$$
[1,-1,0,0,0,0,0,0], \ldots,[0,0,0,0,0,0,1,-1],
$$

there are the 28-hyperplanes

$$
\mathcal{L}_{28_{12}}^{5}=\left\{x_{1}=x_{2}\right\}, \ldots, \mathcal{L}_{2878}^{5}=\left\{x_{7}=x_{8}\right\} .
$$

The involutions

$$
x_{1} \leftrightarrow x_{2}, \ldots, x_{7} \leftrightarrow x_{8}
$$

pointwise fix the respective hyperplanes. These 28 transpositions generate $\mathcal{G}_{8 \text { ! }}$ so that it acts as both a real and complex reflection group. (See Shephard and Todd 1954.) 
Various special planes and lines appear as intersections of the 28-hyperplanes. TABLE 2.2 and TABLE 2.3 summarize the situation. Of particular dynamical significance is the collection of 28-lines $\mathcal{L}_{28_{i j}}^{1}$. This configuration forms the complete graph on the 8-points. (See Figure 1 for two views.)
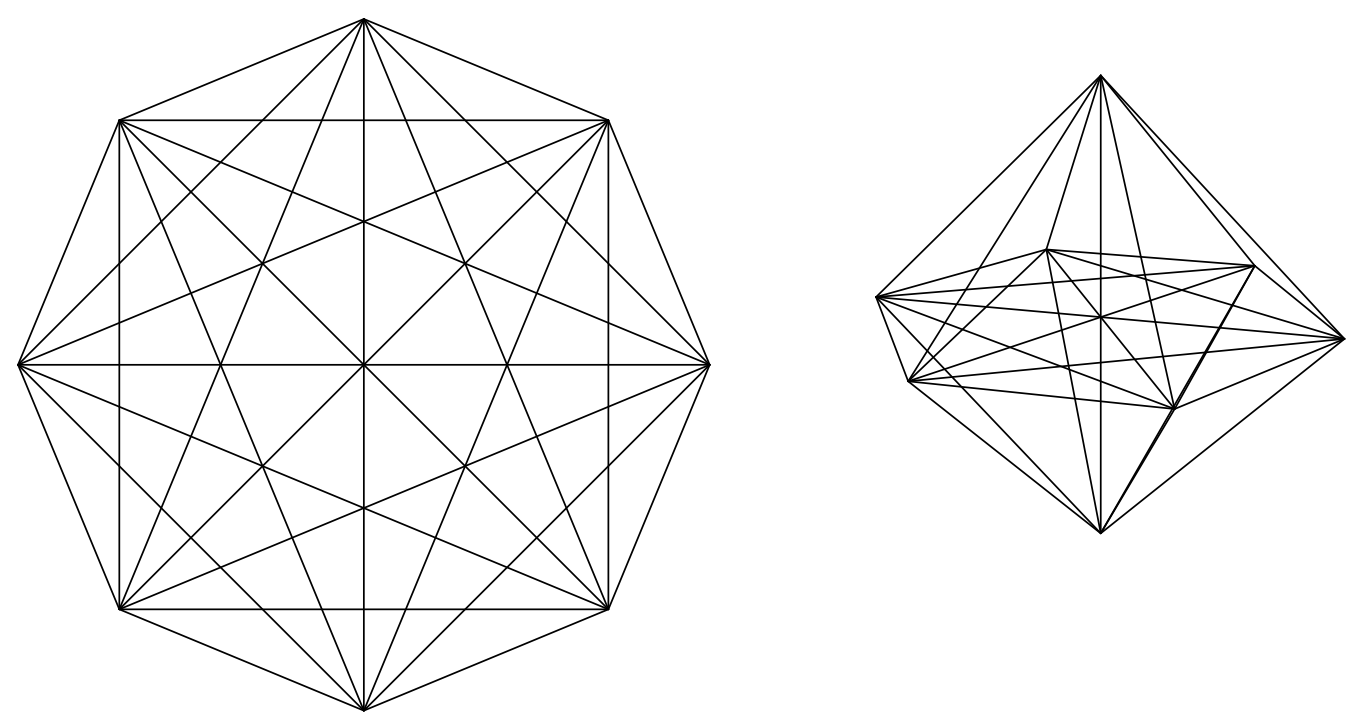

Figure 1. Configuration of 28-lines and 8-points

TABLE 2.1. Three special orbits

\begin{tabular}{c|c|c|c} 
Size & Representative & Descriptor & Stabilizer \\
\hline 8 & {$[-7,1,1,1,1,1,1,1]$} & $p_{1}^{8}$ & $\mathcal{S}_{7}$ \\
28 & {$[0,0,0,0,0,0,1,-1]$} & $p_{78}^{28}$ & $\mathcal{S}_{6} \times \mathbf{Z}_{2}$ \\
28 & {$[1,1,1,1,1,1,-3,-3]$} & $q_{78}^{28}$ & $\mathcal{S}_{6} \times \mathbf{Z}_{2}$
\end{tabular}

\section{EquiVARIANT MAPS}

The primary tool to be used in solving the general octic is a rational map $f$ with $\mathcal{S}_{8}$ symmetry. In algebraic terms, this means that

$$
f \circ T=T f \text { for all } T \in \mathcal{G}_{8} \text { ! }
$$

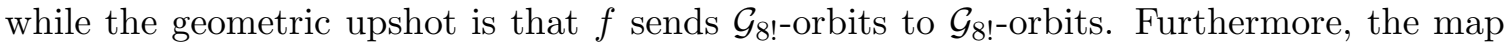
should have reliable dynamics: its attractor

1 ) is a single $\mathcal{G}_{8 \text { ! }}$ orbit

2) has a corresponding basin with full measure in $\mathbf{C P}^{6}$ (strongly reliable)

$\left.2^{\prime}\right)$ alternatively, has a corresponding basin that is dense in $\mathbf{C P}^{6}$ (weakly reliable). 
TABLE 2.2. Some fundamental $\mathbf{C P}^{2}$ orbits

\begin{tabular}{c|c|c|c|c} 
Geometric definition & Descriptor & $\begin{array}{c}\text { Set-wise } \\
\text { stabilizer }\end{array}$ & $\begin{array}{c}\text { Point-wise } \\
\text { stabilizer }\end{array}$ & $\begin{array}{c}\text { Restricted } \\
\text { action }\end{array}$ \\
\hline $\mathcal{L}_{28_{i j}}^{5} \cap \mathcal{L}_{28_{i k}}^{5} \cap \mathcal{L}_{28_{i \ell}}^{5} \cap \mathcal{L}_{28_{i m}}^{5}$ & $\mathcal{L}_{56}^{2}$ & $\mathcal{S}_{5} \times \mathcal{S}_{3}$ & $\mathcal{S}_{5}$ & $\mathcal{S}_{3}$ \\
$\mathcal{L}_{28_{i j}}^{5} \cap \mathcal{L}_{28_{k \ell}}^{5} \cap \mathcal{L}_{28_{m n}}^{5} \cap \mathcal{L}_{28_{p q}}^{5}$ & $\mathcal{L}_{105}^{2}$ & $\mathcal{S}_{4} \times \mathbf{Z}_{2}^{4}$ & $\mathbf{Z}_{2}^{4}$ & $\mathcal{S}_{4}$ \\
$\mathcal{L}_{28_{i j}}^{5} \cap \mathcal{L}_{28_{i k}}^{5} \cap \mathcal{L}_{28_{\ell m}}^{5} \cap \mathcal{L}_{28_{\ell n}}^{5}$ & $\mathcal{L}_{280}^{2}$ & $\mathcal{S}_{3}^{2} \times \mathbf{Z}_{2}^{2}$ & $\mathcal{S}_{3}^{2}$ & $\mathbf{Z}_{2}^{2}$ \\
$\mathcal{L}_{28_{i j}}^{5} \cap \mathcal{L}_{28_{k \ell}}^{5} \cap \mathcal{L}_{28_{k m}}^{5} \cap \mathcal{L}_{28_{k n}}^{5}$ & $\mathcal{L}_{420}^{2}$ & $\mathcal{S}_{4} \times \mathbf{Z}_{2}^{2}$ & $\mathcal{S}_{4} \times \mathbf{Z}_{2}$ & $\mathbf{Z}_{2}$ \\
$\mathcal{L}_{28_{i j}}^{5} \cap \mathcal{L}_{28_{k \ell}}^{5} \cap \mathcal{L}_{28_{m n}}^{5} \cap \mathcal{L}_{28_{m p}}^{5}$ & $\mathcal{L}_{840}^{2}$ & $\mathcal{S}_{3} \times \mathbf{Z}_{2}^{3}$ & $\mathcal{S}_{3} \times \mathbf{Z}_{2}^{2}$ & $\mathbf{Z}_{2}$
\end{tabular}

TABLE 2.3. Special $\mathbf{C P}^{1}$ orbits

\begin{tabular}{c|c|c|c|c} 
Geometric definition & Descriptor & $\begin{array}{c}\text { Set-wise } \\
\text { stabilizer }\end{array}$ & $\begin{array}{c}\text { Point-wise } \\
\text { stabilizer }\end{array}$ & $\begin{array}{c}\text { Restricted } \\
\text { action }\end{array}$ \\
\hline $\mathcal{L}_{28_{i j}}^{5} \cap \mathcal{L}_{28_{i k}}^{5} \cap \mathcal{L}_{28_{i \ell}}^{5} \cap \mathcal{L}_{28_{i m}}^{5} \cap \mathcal{L}_{28_{i n}}^{5}$ & $\mathcal{L}_{28_{p q}}^{1}$ & $\mathcal{S}_{6} \times \mathbf{Z}_{2}$ & $\mathcal{S}_{6}$ & $\mathbf{Z}_{2}$ \\
& & & \\
$\mathcal{L}_{28_{i j}}^{5} \cap \mathcal{L}_{28_{k \ell}, \ldots, n}^{5} \cap \mathcal{L}_{28_{k m}}^{5} \cap \mathcal{L}_{28_{k n}}^{5} \cap \mathcal{L}_{28_{k p}}^{5}$ & $\mathcal{L}_{168}^{1}$ & $\mathcal{S}_{5} \times \mathbf{Z}_{2}$ & $\mathcal{S}_{5} \times \mathbf{Z}_{2}$ & $\mathbf{Z}_{1}$ \\
$\mathcal{L}_{28_{i j}}^{5} \cap \mathcal{L}_{28_{k \ell}}^{5} \cap \mathcal{L}_{28_{m n}}^{5} \cap \mathcal{L}_{28_{m p}}^{5} \cap \mathcal{L}_{28_{m q}}^{5}$ & $\mathcal{L}_{210}^{1}$ & $\mathcal{S}_{4} \times \mathbf{Z}_{2}^{3}$ & $\mathcal{S}_{4} \times \mathbf{Z}_{2}^{2}$ & $\mathbf{Z}_{2}$ \\
$\mathcal{L}_{28_{i j}}^{5} \cap \mathcal{L}_{28_{k \ell}}^{5} \cap \mathcal{L}_{28_{k m}}^{5} \cap \mathcal{L}_{28_{n p}}^{5} \cap \mathcal{L}_{28_{n q}}^{5}$ & $\mathcal{L}_{280}^{1}$ & $\mathcal{S}_{3}^{2} \times \mathbf{Z}_{2}^{2}$ & $\mathcal{S}_{3} \times \mathbf{Z}_{2}$ & $\mathbf{Z}_{2}$ \\
$\mathcal{L}_{28_{i j}}^{5} \cap \mathcal{L}_{28_{i k}}^{5} \cap \mathcal{L}_{28_{\ell m}}^{5} \cap \mathcal{L}_{28_{\ell n}}^{5} \cap \mathcal{L}_{28_{\ell p}}^{5}$ & $\mathcal{M}_{280}^{1}$ & $\mathcal{S}_{4} \times \mathcal{S}_{3}$ & $\mathcal{S}_{4} \times \mathcal{S}_{3}$ & $\mathbf{Z}_{1}$
\end{tabular}

3.1. Basic maps. A finite group action $\mathcal{G}$ on $\mathbf{C}^{n}$ induces an action on the associated exterior algebra. Moreover, $\mathcal{G}$-invariant $(n-1)$-forms correspond to $\mathcal{G}$-equivariant maps. (See Crass 1999.)

For a reflection group, the number of generating 0-forms (that is, polynomials) is the dimension of the action. [Shephard and Todd 1954, p. 282] From a result in complex reflection groups, this is also the number of generating 1-forms and $(n-1)$-forms. Orlik and Terao 1992, p. 232] Indeed, the generating 1 -forms are exterior derivatives of the 0 -forms while the generating $(n-1)$-forms are wedge products of 1 -forms.

Proposition 3.1. With $X_{i}^{k}=-7 x_{i}^{k}+\sum_{j \neq i} x_{j}^{k}$, the seven maps

$$
f_{k}(x)=\left[X_{1}^{k}, X_{2}^{k}, X_{3}^{k}, X_{4}^{k}, X_{5}^{k}, X_{6}^{k}, X_{7}^{k}, X_{8}^{k}\right] \quad k=1, \ldots, 7
$$

generate the module of $\mathcal{G}_{8 \text { ! }}$ equivariants over the ring of $\mathcal{G}_{8 \text { ! }}$ invariants. 
These maps are projections onto the hyperplane $\mathcal{H}_{x}$ along $[1,1,1,1,1,1,1,1]$ of the power maps

$$
\left[x_{1}^{k}, x_{2}^{k}, x_{3}^{k}, x_{4}^{k}, x_{5}^{k}, x_{6}^{k}, x_{7}^{k}, x_{8}^{k}\right] .
$$

Proposition 3.2. Under an orthogonal action an invariant $F(x)$ gives rise to an equivariant $f(x)$ by means of a formal gradient

$$
f(x)=\nabla_{x} F(x)=\left[\frac{\partial F}{\partial x_{1}}(x), \ldots, \frac{\partial F}{\partial x_{n}}(x)\right] .
$$

Proof. See [rass 2001].

Note that the $\mathcal{G}_{8 !}$-equivariant $f_{k}(x)$ is not equal to $\nabla_{x} F_{k+1}(x)$, but is a multiple of

$$
\left.\nabla_{x} F_{k+1}(x)\right|_{x_{i}^{k} \rightarrow X_{i}^{k}} \text {. }
$$

While this may be a source of confusion, it does not cause problems since we are working on the hyperplane $\mathcal{H}_{x}$. When using hyperplane coordinates on $\mathcal{H}_{u}$, the discrepancy manifests itself in the appearance of a constant $-\frac{8}{k+1}$ for each map $\phi_{k}(u)$. (See below.)

Recalling the change of coordinates from $\mathcal{H}_{x}$ to $\mathcal{H}_{u}$, a map on $\mathcal{H}_{x}$ expresses itself as a map

$$
\phi(u)=H f\left(\overline{H^{T}} u\right)
$$

on $\mathcal{H}_{u}$. Having these maps in terms of the basic $u$-invariants $\Phi_{k}(u)$ will be useful.

Definition 3.1. Let

$$
R=\left(r_{i j}\right)=\left\{\begin{array}{ll}
1 & i+j=8 \\
0 & \text { otherwise }
\end{array} \text { and } \nabla_{u}^{r} F(u)=R \nabla_{u} F(u)\right.
$$

represent the reversed identity and reversed gradient.

Proposition 3.3. In $\mathcal{H}_{u}$ coordinates, the map $\phi(u)=H f\left(\overline{H^{T}} u\right)$ is given by

$$
\phi(u)=\nabla_{u}^{r} \Phi(u)
$$

where $\Phi(u)=F\left(\overline{H^{T}} u\right)=F(x)$ and $f(x)=\nabla_{x} F(x)$.

Proof. Crass 2001.

Thus, the generating equivariants $\phi_{k}(u)=H f_{k}\left(\overline{H^{T}} u\right)$ are

$$
\phi_{k}(u)=-\frac{8}{k+1} \nabla_{u}^{r} \Phi_{k+1}(u) .
$$

Although the factors $-\frac{8}{k+1}$ have no projective effect on the maps individually, they do play a role when forming combinations of maps from parametrized families that do not have a common degree. (See Section 4.6.) Explicit expressions for the maps of degrees one and two are

$$
\begin{aligned}
\phi_{1}(u)= & -8\left[u_{1}, u_{2}, u_{3}, u_{4}, u_{5}, u_{6}, u_{7}\right] \\
\phi_{2}(u)= & -2 \sqrt{2}\left[2\left(u_{4} u_{5}+u_{3} u_{6}+u_{2} u_{7}\right), u_{1}^{2}+u_{5}^{2}+2 u_{4} u_{6}+2 u_{3} u_{7},\right. \\
& 2\left(u_{1} u_{2}+u_{5} u_{6}+u_{4} u_{7}\right), u_{2}^{2}+2 u_{1} u_{3}+u_{6}^{2}+2 u_{5} u_{7}, 2\left(u_{2} u_{3}+u_{1} u_{4}+u_{6} u_{7}\right), \\
& \left.u_{3}^{2}+2 u_{2} u_{4}+2 u_{1} u_{5}+u_{7}^{2}, 2\left(u_{3} u_{4}+u_{2} u_{5}+u_{1} u_{6}\right)\right] .
\end{aligned}
$$

The lengthy results for the remaining maps are available at Crass 2000. 
3.2. A fixed point property. For a $\mathcal{G}_{8 !}$-equivariant $f$ and a point $a$ that an element $T \in \mathcal{G}_{8 !}$ fixes,

$$
T f(a)=f(T a)=f(a) .
$$

Hence, equivariants preserve fixed points of a group element.

Being pointwise fixed by the involution

$$
x_{i} \longleftrightarrow x_{j}
$$

a 28-hyperplane

$$
\mathcal{L}_{28_{i j}}^{5}=\left\{x_{i}-x_{j}=0\right\}
$$

either maps to itself or collapses to its companion 28-point

$$
p_{i j}^{28}=[\ldots 0 \ldots, \overbrace{1}^{i}, \ldots 0 \ldots, \overbrace{-1}^{j}, \ldots 0 \ldots] \notin \mathcal{L}_{28_{i j}}^{5} .
$$

In the former generic case, the map preserves the planes and lines that are intersections of 28-hyperplanes. (See Tables 2.2 and 2.3.)

3.3. Families of equivariants. The $\mathcal{G}_{8}$ ! equivariants form a degree-graded module over the $\mathcal{G}_{8}$ ! invariants. This means that for an invariant $F_{\ell}$ and equivariant $g_{m}$ of degrees $\ell$ and $m$, the product

$$
F_{\ell} \cdot g_{m}
$$

is an equivariant of degree $\ell+m$. When looking for a map in a certain degree $k$ with special geometric or dynamical properties, my approach is to express the entire family of " $k$-maps" and by manipulation of parameters, locate a subfamily with the desired behavior.

3.4. A special map in degree four. In the configuration of 28-lines $\mathcal{L}_{28_{i j}}^{1}$ each 8-point lies at the intersection of seven lines while each $\mathcal{L}_{28_{i j}}^{1}$ contains the points $p_{i}^{8}$ and $p_{j}^{8}$. (See Section 2.3.) Moreover, these are the only intersections of 28-lines. We can attempt to exploit this structure by looking for a map with superattracting "pipes" along the 28-lines: this means that, at each point on the line, the map is critical in all five "off-line" directions. Under such a map, the 8-points would be superattracting in all directions. In addition, we want the map's degree to be as small as possible. Degrees two and three avail us of too few parameters.

The family of 4-maps has (homogeneous) dimension three:

$$
\alpha_{1} \Phi_{3} \phi_{1}+\alpha_{2} \Phi_{2} \phi_{2}+\alpha_{3} \phi_{4}
$$

Of course, choosing two parameter values determines a map on projective space. Obtaining a map $g_{4}$ for which the 28-lines are critical in the off-line directions requires two parameters. With the third parameter, we choose a lift of the map to $\mathbf{C}^{7}$ that fixes the 8-points. The result is

$$
g_{4}=-2 \Phi_{3} \phi_{1}-9 \Phi_{2} \phi_{2}+84 \phi_{4} .
$$

The central dynamical role played by the 8-points suggests that a good choice of coordinates for this map places these points at

$$
[1,0,0,0,0,0,0], \ldots,[0,0,0,0,0,0,1],[1,1,1,1,1,1,1] .
$$

Using $\left[v_{1}, \ldots, v_{7}\right]$ for this system, the map takes the form

$$
g_{4}(v)=\left[v_{1} T_{1}(v), \ldots, v_{7} T_{7}(v)\right]
$$


where

$$
T_{k}(v)=7 v_{k}^{3}-4 v_{k}^{2} S_{1}\left(\widehat{v_{k}}\right)+2 v_{k} S_{2}\left(\widehat{v_{k}}\right)-S_{3}\left(\widehat{v_{k}}\right),
$$

$S_{k}$ is the degree- $k$ elementary symmetric function in six variables, and

$$
\widehat{v_{k}}=\left(\ldots, v_{k-1}, v_{k+1}, \ldots\right) .
$$

In the $v$-coordinates, the equations

$$
v_{i}=x, v_{j}=y, v_{k}=0 \quad \text { for } k \neq i, j
$$

determine 21 of the 28 -lines $\mathcal{L}_{28_{i j}}^{1}$. For all $k$, each term in $S_{2}\left(\widehat{v_{k}}\right)$ and $S_{3}\left(\widehat{v_{k}}\right)$ contains at least one $v_{\ell}(\ell \neq i, j)$. Thus,

$$
\left.S_{2}\left(\widehat{v_{k}}\right)\right|_{\mathcal{L}_{28_{i j}}^{1}}=0 \quad \text { and }\left.\quad S_{3}\left(\widehat{v_{k}}\right)\right|_{\mathcal{L}_{28_{i j}}^{1}}=0
$$

so that in the inhomogeneous coordinate $z=\frac{x}{y}$ on the line the map restricts to

$$
z \longrightarrow-z^{3} \frac{7 z-4}{4 z-7}
$$

while the pair of 8 -points appear at 0 and $\infty$.

Proposition 3.4. The 8-points $p_{i}^{8}$, $p_{j}^{8}$ are the attractor for the restriction $g=\left.g_{4}\right|_{\mathcal{L}_{28_{i j}}^{1}}$. Furthermore, in the coordinates used above, the Julia set $J_{g}$ is the unit circle.

Proof. Since the mobius transformation

$$
\frac{7 z-4}{4 z-7}
$$

preserves the unit disk,

$$
|g(z)|<|z|^{3}
$$

for $|z|<1$. If $|z|<1$, iteration yields

$$
\left|g^{n}(z)\right|<\left|g^{n-1}(z)\right|^{3}<\cdots<|g(z)|^{3^{n-1}}<|z|^{3^{n}} .
$$

Thus, every point in the disk belongs to the basin of the superattracting fixed point 0 . Since $g$ is symmetric under

$$
z \longrightarrow \frac{1}{z}
$$

the basin of $\infty$ is $\{|z|>1\}$.

The complete invariance of $\{|z|=1\}$ implies that it contains and, indeed, is the map's Julia set.

Thus, the basins of the 8-points contain the 28-lines excepting the $\mathbf{R P}^{1}$ equator - the unit circle in the coordinates above. Along this circle $C, g_{4}$ has periodic, preperiodic, and what we might call chaotic saddle points. Attached to each point $z$ on $C$ is a 5 -dimensional stable manifold $W_{z}$ consisting of points attracted to $C$, that is, to the trajectory of $z$. Locally, the stable manifolds are mutually disjoint and, collectively over the circle, give a stable manifold $W_{C}$ of $C$ whose real-dimension is eleven and that belongs to the basin boundaries of the pair of 8-points. We can see (real) 2-dimensional slices of this stable manifold by plotting basins of attraction on spaces that are $g_{4}$-invariant and intersect $W_{C}$. (See Appendix 6.) 
By construction, $g_{4}$ self-maps each $\mathcal{S}_{6}$-symmetric 28-hyperplane $\mathcal{L}_{28_{i j}}^{5}$ and hence, preserves each $\mathbf{C P}^{1}$ and $\mathbf{C P}^{2}$ intersection of hyperplanes. Denote these 1- and 2-dimensional spaces by $\mathcal{L}_{m}^{1}$ and $\mathcal{L}_{n}^{2}$. Furthermore, $g_{4}$ is equivariant under the antiholomorphic transformation

$$
x \longrightarrow \bar{x}
$$

and, thereby, preserves $\mathcal{R}$ - the $\mathcal{S}_{8}$-symmetric $\mathbf{R P}^{6}$. We can get a picture of the map's restricted dynamics by plotting basins of attraction on $\mathcal{L}_{m}^{1}-\mathrm{a} \mathbf{C P}^{1}$-and on the $\mathbf{R P}^{2}$ intersections

$$
\mathcal{L}_{n}^{2} \cap \mathcal{R}
$$

The basin portraits appear in ApPEndix 6. Graphical and experimental evidence support a claim of reliability for $g_{4}$.

Conjecture 3.1. The attractor for $g_{4}$ is the 8-point orbit.

Conjecture 3.2. Under $g_{4}$, the basins of the 8-points fill up $\mathbf{C P}^{6}$ in measure.

Finally, since $g_{4}$ has real coefficients (see (3.3)), it preserves the $\mathbf{R P}^{6}$ whose points have real $u$ coordinates. This is not the $\mathcal{S}_{8}$-symmetric $\mathcal{R}$. Rather it has the $\mathcal{S}_{7}$ symmetry of $p_{1}^{8}$ which is $[1,1,1,1,1,1,1]$ in the $u$ space. Accordingly, there are eight spaces of this type.

\section{Solving the octic}

To compute a root of a polynomial, one must overcome its symmetry. For a general equation of degree $n$ the obstacle is $\mathcal{S}_{n}$. Klein described a means to this end: given values for an independent set of $\mathcal{S}_{n}$-invariant homogeneous polynomials

$$
a_{1}=G_{1}(x) \ldots a_{m}=G_{m}(x),
$$

find the $\mathcal{S}_{n}$ orbits of solutions $x$ to these equations. [Klein 1913, pp. 69ff] This task of inverting the $G_{k}$ is the form problem on $\mathcal{S}_{n}$. It also has an inhomogeneous manifestation: for $m-1$ given values, invert $m-1$ invariant rational functions of degree zero.

By iterating a reliable $\mathcal{S}_{n}$ equivariant we can break the obstructing symmetry. In effect, the dynamics provides a mechanism for solving the form problem and, hence, the $n$th degree equation.

4.1. Parameters. The $\mathcal{G}_{8 \text { ! }}$ rational form problem is to solve

$$
\begin{aligned}
& K_{1}=\frac{\Phi_{3}(u)^{2}}{\Phi_{2}(u)^{3}} \quad K_{2}=\frac{\Phi_{4}(u)}{\Phi_{2}(u)^{2}} \\
& K_{3}=\frac{\Phi_{5}(u)}{\Phi_{2}(u) \Phi_{3}(u)} \\
& K_{4}=\frac{\Phi_{6}(u)}{\Phi_{2}(u)^{3}} \\
& K_{5}=\frac{\Phi_{7}(u)}{\Phi_{2}(u) \Phi_{5}(u)} \\
& K_{6}=\frac{\Phi_{8}(u)}{\Phi_{2}(u)^{4}} \text {. }
\end{aligned}
$$

As functions, the $K_{i}$ define the $\mathcal{G}_{8 \text { ! }}$ quotient map

$$
\begin{aligned}
& {\left[K_{1}, K_{2}, K_{3}, K_{4}, K_{5}, K_{6}, 1\right]=} \\
& \quad\left[\Phi_{2} \Phi_{3}^{3} \Phi_{5}, \Phi_{2}^{2} \Phi_{3} \Phi_{4} \Phi_{5}, \Phi_{2}^{3} \Phi_{5}^{2}, \Phi_{2} \Phi_{3} \Phi_{5} \Phi_{6}, \Phi_{2}^{3} \Phi_{3} \Phi_{7}, \Phi_{3} \Phi_{5} \Phi_{8}, \Phi_{2}^{4} \Phi_{3} \Phi_{5}\right]
\end{aligned}
$$

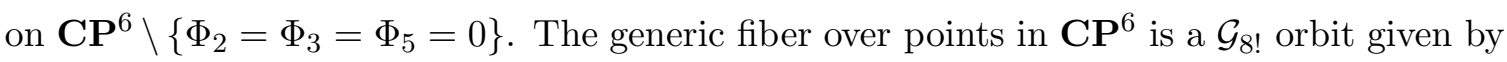

$$
\begin{aligned}
& \left\{\Phi_{3}^{2}=K_{1} \Phi_{2}^{3}\right\} \cap\left\{\Phi_{4}=K_{2} \Phi_{2}^{2}\right\} \cap\left\{\Phi_{5}=K_{3} \Phi_{2} \Phi_{3}\right\} \\
& \cap\left\{\Phi_{6}=K_{4} \Phi_{2}^{3}\right\} \cap\left\{\Phi_{7}=K_{5} \Phi_{2} \Phi_{5}\right\} \cap\left\{\Phi_{8}=K_{6} \Phi_{2}^{4}\right\} .
\end{aligned}
$$


Exceptional locations are

$$
[0,0,1,0,0,0,0],[0,0,0,0,1,0,0],[0,0,0,0,0,1,0]
$$

where the respective fibers are the hypersurfaces $\left\{\Phi_{3}=0\right\},\left\{\Phi_{5}=0\right\}$, and $\left\{\Phi_{2}=0\right\}$. The parameters $K_{i}$ forge a link between octic equations and $\mathcal{G}_{8 \text { ! }}$ actions. The connection consists in $K$-parametrizations of each regime.

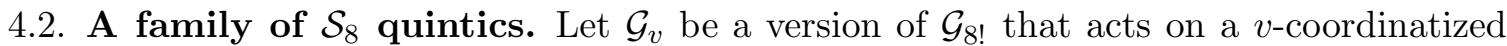
$\mathbf{C P}_{v}^{6}$. This will be a parameter space - the coordinate $v$ merely stands in for $u$. The linear polynomials

$$
X_{k}(x)=-7 x_{k}+\sum_{i \neq k} x_{i}
$$

form an orbit of size eight. Let

$$
L_{k}(u)=X_{k}\left(\overline{H^{T}} u\right)
$$

Then the rational functions

$$
\sigma_{k}(v)=\frac{\Phi_{2}(v) L_{k}(v)}{\Phi_{3}(v)}
$$

also give an 8-orbit. Taking the $\sigma_{k}$ as roots of a polynomial

$$
R_{v}(s)=\prod_{k=1}^{8}\left(s-\sigma_{k}(v)\right)=\sum_{k=0}^{8} C_{k}(v) s^{8-k}
$$

creates a family of octics whose members generically have $\mathcal{S}_{8}$ symmetry. Since $\mathcal{G}_{v}$ permutes the $\sigma_{k}(v)$, each coefficient $C_{k}(v)$ is $\mathcal{G}_{v}$-invariant and hence, expressible in the basic forms $\Phi_{k}(v)$. Ultimately, we can express each $C_{k}$ in terms of the $K_{i}$. By direct calculation,

$$
\begin{aligned}
& C_{0}=1 \\
& C_{1}=0 \\
& C_{2}=-\frac{32}{K_{1}} \\
& C_{3}=\frac{512}{3 K_{1}^{2}} \\
& C_{4}=\frac{512\left(1-2 K_{2}\right)}{8 K_{1}^{2}} \\
& C_{5}=\frac{16384\left(-5+6 K_{3}\right)}{15 K_{1}^{2}} \\
& C_{6}=\frac{-16384\left(-3+8 K_{1}+18 K_{2}-24 K_{4}\right)}{9 K_{1}^{3}} \\
& C_{7}=\frac{262144\left(35-70 K_{2}-84 K_{3}-120 K_{3} K_{5}\right)}{105 K_{1}^{3}} \\
& C_{8}=\frac{131072\left(15-160 K_{1}+180 K_{2}+180 K_{2}^{2}+384 K_{1} K_{3}+480 K_{4}-720 K_{6}\right)}{45 K_{1}^{4}} .
\end{aligned}
$$


Members of the family of octic resolvents

$$
R_{K}(s)=\sum_{n=0}^{8} C_{n} s^{8-n}
$$

parametrized by $K=\left(K_{1}, \ldots, K_{6}\right)$ are particularly well-suited for an iterative solution that employs $g_{4}$. For chosen values of the $K_{i}$, a solution to the resulting form problem yields a

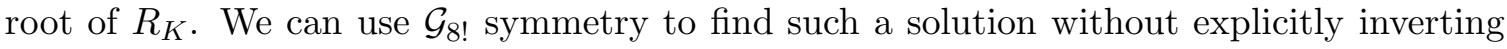
the $K_{i}$ equations. Our attention will turn to this issue after we connect the general octic to the special family $R_{K}$.

4.3. Reduction of the general octic to the $\mathcal{G}_{8 !}$ resolvent. By means of a linear Tschirnhaus transformation

the general octic

$$
x \longrightarrow y-\frac{a_{1}}{8}
$$

$$
p(x)=x^{8}+a_{1} x^{7}+a_{2} x^{6}+a_{3} x^{5}+a_{4} x^{4}+a_{5} x^{3}+a_{6} x^{2}+a_{7} x+a_{8}
$$

becomes the standard 7-parameter resolvent

$$
q(y)=y^{8}+b_{2} y^{6}+b_{3} y^{5}+b_{4} y^{4}+b_{5} y^{3}+b_{6} y^{2}+b_{7} y+b_{8}
$$

where

$$
\begin{aligned}
b_{2}= & \frac{-7 a_{1}^{2}+16 a_{2}}{16} \\
b_{3}= & \frac{7 a_{1}^{3}-24 a_{1} a_{2}+32 a_{3}}{32} \\
b_{4}= & \frac{-105 a_{1}^{4}+480 a_{1}^{2} a_{2}-1280 a_{1} a_{3}+2048 a_{4}}{2048} \\
b_{5}= & \frac{7 a_{1}^{5}-40 a_{1}^{3} a_{2}+160 a_{1}^{2} a_{3}-512 a_{1} a_{4}+1024 a_{5}}{1024} \\
b_{6}= & \frac{-35 a_{1}^{6}+240 a_{1}^{4} a_{2}-1280 a_{1}^{3} a_{3}+6144 a_{1}^{2} a_{4}-24576 a_{1} a_{5}+65536 a_{6}}{65536} \\
b_{7}= & \frac{3 a_{1}^{7}-24 a_{1}^{5} a_{2}+160 a_{1}^{4} a_{3}-1024 a_{1}^{3} a_{4}+6144 a_{1}^{2} a_{5}-32768 a_{1} a_{6}+131072 a_{7}}{131072} \\
b_{8}= & \frac{-7}{16777216}\left(a_{1}^{8}+64 a_{1}^{6} a_{2}-512 a_{1}^{5} a_{3}+4096 a_{1}^{4} a_{4}-32768 a_{1}^{3} a_{5}+262144 a_{1}^{2} a_{6}\right. \\
& \left.-2097152 a_{1} a_{7}+16777216 a_{8}\right) .
\end{aligned}
$$

Application of another linear Tschirnhaus transformation

$$
s \longrightarrow \frac{y}{\lambda}
$$

converts the 6-parameter family $R_{K}(s)$ into a $\mathcal{G}_{8 \text { ! }}$ resolvent

$$
\Sigma_{K, \lambda}(y)=\lambda^{8} R_{K}\left(\frac{y}{\lambda}\right)=\sum_{n=0}^{8} \lambda^{n} C_{n} y^{8-n}
$$

in the seven parameters $K_{1}, \ldots, K_{6}$, and the auxiliary $\lambda$.

The functions

$$
b_{n}=\lambda^{n} C_{n}(K)
$$


relate the coefficients of $q$ and $\Sigma_{K, \lambda}$. The $b_{n}$ invert to

$$
\begin{aligned}
K_{1} & =\frac{-9 b_{3}^{2}}{8 b_{2}^{3}} \\
K_{2} & =\frac{b_{2}^{2}-2 b_{4}}{2 b_{2}^{2}} \\
K_{3} & =\frac{5\left(b_{2} b_{3}-b_{5}\right)}{6 b_{2} b_{3}} \\
K_{4} & =\frac{2 b_{2}^{3}-3 b_{3}^{2}-6 b_{2} b_{4}+6 b_{6}}{8 b_{2}^{3}} \\
K_{5} & =\frac{7\left(b_{2}^{2} b_{3}-b_{3} b_{4}-b_{2} b_{5}+b_{7}\right)}{10 b_{2}\left(b_{2} b_{3}-b_{5}\right)} \\
K_{6} & =\frac{b_{2}^{4}-4 b_{2} b_{3}^{2}-4 b_{2}^{2} b_{4}+2 b_{4}^{2}+4 b_{3} b_{5}+4 b_{2} b_{6}-4 b_{8}}{8 b_{2}^{4}} \\
\lambda & =\frac{-3 b_{3}}{16 b_{2}} .
\end{aligned}
$$

Thus, almost any octic descends to a member of $R_{K}$. The reduction fails when

$$
-7 a_{1}^{2}+16 a_{2}=16 b_{2}=0 \quad \text { or } \quad 7 a_{1}^{3}-24 a_{1} a_{2}+32 a_{3}=32 b_{3}=0
$$

or

$$
\begin{aligned}
& -105 a_{1}^{5}+600 a_{1}^{3} a_{2}-768 a_{1} a_{2}^{2}-608 a_{1}^{2} a_{3}+1024 a_{2} a_{3}+512 a_{1} a_{4}-1024 a_{5} \\
& =1024\left(b_{2} b_{3}-b_{5}\right)=0 .
\end{aligned}
$$

A solution to the special resolvent $R_{K}$ then ascends to a solution to the general quintic.

4.4. A family of $\mathcal{S}_{8}$ actions. With the basic $\mathcal{G}_{v}$ forms and maps, we can define the parametrized change of coordinates

$$
u=\tau_{v} w=\sum_{k=1}^{7}\left(\Phi_{9-k}(v) \phi_{k}(v)\right) w_{k} .
$$

For a choice of parameter $v$,

$$
\tau_{v}: \mathbf{C P}_{w}^{6} \longrightarrow \mathbf{C P}_{u}^{6}
$$

is linear in $w$ and provides a parametrized family of $\mathcal{G}_{8}$ groups

$$
\mathcal{G}_{w}^{v}=\tau_{v}^{-1} \mathcal{G}_{u} \tau_{v} .
$$

A matrix form results from taking the $\phi_{k}(v)$ as column vectors:

$$
\left(\begin{array}{c}
u_{1} \\
\vdots \\
u_{7}
\end{array}\right)=\left(\begin{array}{lll}
\Phi_{8}(v) \phi_{1}(v) & \ldots & \Phi_{2}(v) \phi_{7}(v)
\end{array}\right)\left(\begin{array}{c}
w_{1} \\
\vdots \\
w_{7}
\end{array}\right) .
$$

The setup here is as follows.

- $\mathcal{G}_{u}$ is a version of $\mathcal{G}_{8 !}$ that acts on a reference space $\mathbf{C P}_{u}^{6}$.

- $\mathcal{G}_{v}$ is a version of $\mathcal{G}_{8 \text { ! }}$ that acts on a parameter space $\mathbf{C P}_{v}^{6}$.

- $\mathcal{G}_{u}$ and $\mathcal{G}_{v}$ have identical expressions in their respective coordinates. 
- $\mathcal{G}_{w}^{v}$ are versions of $\mathcal{G}_{8 !}$ that act on v-parametrized spaces $\mathbf{C P}_{w}^{6}$.

- The iteration that solves octics $R_{K}$ takes place in $\mathbf{C P}_{w}^{6}$.

Each $\mathcal{G}_{w}^{v}$ has its system of invariants and equivariants. From this point of view we can see, in the resolvents $R_{v}$ and $\mathcal{G}_{w}^{v}$ equivariants, a connection between octics and dynamical systems. Furthermore, each $\mathcal{G}_{w}^{v}$ invariant and equivariant is expressible in the $K_{i}$. This circumstance connects a resolvent $R_{K}$ with $\mathcal{G}_{w}^{v}$-symmetric maps.

By construction, $\tau_{v} w$ possesses an equivariance property:

$$
\tau_{A v} w=A \tau_{v} w \quad \text { for } A \in \mathcal{G}_{v}, \mathcal{G}_{u}
$$

The determinant of $\tau_{v}$ will enter into upcoming calculations and so, demands some attention. Since

$$
\operatorname{det} \tau_{A v}=\operatorname{det} A \operatorname{det} \tau_{v}
$$

$\operatorname{det} \tau_{v}$ is invariant under the $\mathcal{A}_{8}$ subgroup $\mathcal{G}_{8 ! / 2}$ of $\mathcal{G}_{v}$ but only relatively invariant under the full $\mathcal{S}_{8}$ group $\mathcal{G}_{8 !}$. (The "even transformations" have determinant 1 while the odd elements have determinant -1.) Furthermore,

$$
\begin{aligned}
\operatorname{det} \tau_{v} & =\left(\prod_{k=2}^{8} \Phi_{k}(v)\right) \operatorname{det}\left(\phi_{1}(v) \cdots \phi_{7}(v)\right) \\
& =\left(\prod_{k=2}^{8} \Phi_{k}(v)\right) \Psi_{28}(v)
\end{aligned}
$$

where $\Psi_{28}$ is, according to a basic result in reflection group theory, a scalar multiple of the product of the 28 linear forms associated with the 28 hyperplanes that are fixed by the reflections that generate $\mathcal{G}_{v}$. Furthermore, $\Psi_{28}$ is invariant under the group $\mathcal{G}_{8 ! / 2}$ (isomorphic to the alternating group $\mathcal{A}_{8}$ ) but is relatively invariant under $\mathcal{G}_{8 !}$. Consequently, the degree126 square of $\operatorname{det} \tau_{v}$ is $\mathcal{G}_{8 !}$-invariant with $K$-expression

$$
\left(\operatorname{det} \tau_{v}\right)^{2}=\Phi_{2}^{63}(v) t_{K}
$$

The explicit form of $t_{K}$ appears at Crass 2000.

4.5. A family of $\mathcal{S}_{8}$ invariants. The equivariance in $v$ of $\tau_{v} w$ implies that $\Phi_{2}\left(\tau_{v} w\right)$ is $\mathcal{G}_{v}$-invariant. Thus, each $w$ coefficient of $\Phi_{2}\left(\tau_{v} w\right)$ inherits the same invariance. Since

$$
\operatorname{deg}_{v} \Phi_{2}\left(\tau_{v} w\right)=\operatorname{deg}_{u} \Phi_{2}(u) \cdot \operatorname{deg}_{v} \tau_{v} w=2 \cdot 9=18
$$

the rational function

$$
\frac{\Phi_{2}(u)}{\Phi_{2}(v)^{9}}=\frac{\Phi_{2}\left(\tau_{v} w\right)}{\Phi_{2}(v)^{9}}
$$

is degree zero in $v$ and thereby, expressible in $K$. For each $w$ monomial, we can solve a system of linear equations whose dimension is that of the degree-18 $\mathcal{G}_{v}$ invariants. The result is an explicit expression in $K$ for each $w$-coefficient of $\Phi_{2}\left(\tau_{v} w\right)$. Let

$$
\Phi_{2}(v)^{9} \Phi_{2_{K}}(w)=\Phi_{2}(u)
$$

define the basic degree- $2 \mathcal{G}_{w}^{v}$ invariant $\Phi_{2_{K}}(w)$. Similar considerations apply in degree three where

$$
\Phi_{2}(v)^{12} \Phi_{3}(v) \Phi_{3_{K}}(w)=\Phi_{3}(u)
$$

The results appear at Crass 2000]. 
By Proposition 2.2, the degree- 4 and degree- 5 invariants derive from those in degrees two and three. The chain rule determines a transformation formula for the bordered hessian. Let $|\cdot|$ represent the determinant and $A^{T}$ the transpose of $A$.

Proposition 4.1. For $y=A x$,

$$
B H_{x}(F(y), G(y), J(y))=\left(\begin{array}{cc}
A^{T} & 0 \\
0 & 1
\end{array}\right) B H_{y}(F(y), G(y), J(y))\left(\begin{array}{cc}
A & 0 \\
0 & 1
\end{array}\right)
$$

where the subscript indicates the variable of differentiation. Thus,

$$
\left|B H_{x}(F(y), G(y), J(y))\right|=|A|^{2}\left|B H_{y}(F(y), G(y), J(y))\right| .
$$

Applied to the parametrized change of variable $w=\tau_{v}^{-1} u$, this formula along with (4.5) and (4.6) yields

$$
\begin{aligned}
G_{4}(u)= & \left|B H_{u}\left(\Phi_{2}(u), \Phi_{3}(u), \Phi_{3}(u)\right)\right| \\
= & \left|B H_{u}\left(\Phi_{2}(v)^{9} \Phi_{2_{K}}(w), \Phi_{2}(v)^{12} \Phi_{3}(v) \Phi_{3_{K}}(w), \Phi_{2}(v)^{12} \Phi_{3}(v) \Phi_{3_{K}}(w)\right)\right| \\
= & \frac{\Phi_{2}(v)^{6 \cdot 9+2 \cdot 12} \Phi_{3}(v)^{2}}{\left|\tau_{v}\right|^{2}}\left|B H_{w}\left(\Phi_{2_{K}}(w), \Phi_{3_{K}}(w), \Phi_{3_{K}}(w)\right)\right| \\
= & \frac{\Phi_{2}(v)^{78} \Phi_{3}(v)^{2}}{\Phi_{2}(v)^{63} t_{K}}\left|B H_{w}\left(\Phi_{2_{K}}(w), \Phi_{3_{K}}(w), \Phi_{3_{K}}(w)\right)\right| \\
= & \frac{\Phi_{2}(v)^{15} \Phi_{3}(v)^{2}}{t_{K}}\left|B H_{w}\left(\Phi_{2_{K}}(w), \Phi_{3_{K}}(w), \Phi_{3_{K}}(w)\right)\right| \\
= & \frac{\Phi_{2}(v)^{18}}{t_{K}} \frac{\Phi_{3}(v)^{2}}{\Phi_{2}(v)^{3}}\left|B H_{w}\left(\Phi_{2_{K}}(w), \Phi_{3_{K}}(w), \Phi_{3_{K}}(w)\right)\right| \\
= & \Phi_{2}(v)^{18} \frac{K_{1}}{t_{K}}\left|B H_{w}\left(\Phi_{2_{K}}(w), \Phi_{3_{K}}(w), \Phi_{3_{K}}(w)\right)\right| \\
= & \Phi_{2}(v)^{18} G_{4_{K}}(w) .
\end{aligned}
$$

Using Proposition 2.2 we obtain

$$
\begin{aligned}
\Phi_{4}(u) & =\frac{1}{576}\left(72 \Phi_{2}(v)^{18} \Phi_{2_{K}}(w)^{2}+\Phi_{2}(v)^{18} G_{4_{K}}(w)\right) \\
& =\Phi_{2}(v)^{18} \Phi_{4_{K}}(w)
\end{aligned}
$$

so that

$$
\begin{aligned}
G_{5}(u) & =\left|B H_{u}\left(\Phi_{2}(u), \Phi_{3}(u), \Phi_{4}(u)\right)\right| \\
& =\left|B H_{u}\left(\Phi_{2}(v)^{9} \Phi_{2_{K}}(w), \Phi_{2}(v)^{12} \Phi_{3}(v) \Phi_{3_{K}}(w), \Phi_{2}(v)^{18} \Phi_{4_{K}}(w)\right)\right| \\
& =\Phi_{2}(v)^{21} \Phi_{3}(v) G_{5_{K}}(w)
\end{aligned}
$$

and

$$
\begin{aligned}
\Phi_{5}(u) & =\frac{1}{768}\left(96 \Phi_{2}(v)^{9} \Phi_{2_{K}}(w) \Phi_{2}(v)^{12} \Phi_{3}(v) \Phi_{3_{K}}(w)+\Phi_{2}(v)^{21} \Phi_{3}(v) G_{5_{K}}(w)\right) \\
& =\Phi_{2}(v)^{21} \Phi_{3}(v) \Phi_{5_{K}}(w)
\end{aligned}
$$


Employed here are the obvious definitions

$$
\begin{aligned}
& G_{4_{K}}(w)=\frac{K_{1}}{t_{K}}\left|B H_{w}\left(\Phi_{2_{K}}(w), \Phi_{3_{K}}(w), \Phi_{3_{K}}(w)\right)\right| \\
& G_{5_{K}}(w)=\frac{1}{t_{K}}\left|B H_{w}\left(\Phi_{2_{K}}(w), \Phi_{3_{K}}(w), \Phi_{4_{K}}(w)\right)\right|
\end{aligned}
$$

as well as natural definitions for $\Phi_{4_{K}}(w)$ and $\Phi_{5_{K}}(w)$.

4.6. A family of $\mathcal{S}_{8}$ equivariants. Emerging from each $\mathcal{G}_{w}^{v}$ action is a version $\tau_{v}^{-1} g_{4}\left(\tau_{v} w\right)$ of $g_{4}(u)$. Being $\mathcal{G}_{v}$-invariant, these maps also admit parametrization by $K$. In this way, each octic $R_{K}$ enters into association with a dynamical system $g_{K}$ on $\mathbf{C P}_{w}^{6}$.

The reversed identity $R$ and gradient $\nabla^{r}=R \nabla$ appeared in the context of a change from eight $x$ coordinates to seven $u$ coordinates. (See Definition 3.1.) In the present setting, a reversed transpose arises.

Definition 4.1. The repose $A^{r}$ of an $n \times n$ matrix $A$ is its reflection through the reversed diagonal - the entries whose subscripts sum to $n+1$. Alternatively,

$$
A^{r}=R A^{T} R \text {. }
$$

Proposition 4.2. For a coordinate change $x=A y$ and a function $F(y)=\tilde{F}(x)$, the reversed gradient map transforms by

$$
\nabla_{y}^{r} F(y)=A^{r} \nabla_{x}^{r} \tilde{F}(x) .
$$

Proof. See [Crass 2001], Proposition 4.2, but note that the coordinate change there should be $w=A u$.

Using (3.2), the degree-1 $\mathcal{G}_{8 \text { ! map is }}$

$$
\begin{aligned}
\phi_{1}(u) & =-\frac{8}{2} \nabla_{u}^{r} \Phi_{2}(v)^{9} \Phi_{2_{K}}(w) \\
& =-4 \Phi_{2}(v)^{9}\left(\tau_{v}^{-1}\right)^{r} \nabla_{w}^{r} \Phi_{2_{K}}(w) \\
& =-4 \Phi_{2}(v)^{9} \tau_{v} \tau_{v}^{-1}\left(\tau_{v}^{-1}\right)^{r} \nabla_{w}^{r} \Phi_{2_{K}}(w) \\
& =-4 \tau_{v} \Phi_{2}(v)^{9}\left(\tau_{v}^{r} \tau_{v}\right)^{-1} \nabla_{w}^{r} \Phi_{2_{K}}(w) .
\end{aligned}
$$

Thus,

$$
\tau_{v}^{-1} \phi_{1}\left(\tau_{v} w\right)=-4 \Phi_{2}(v)^{9}\left(\tau_{v}^{r} \tau_{v}\right)^{-1} \nabla_{w}^{r} \Phi_{3_{K}}(w) .
$$

Using the description on the left-hand side, a straightforward calculation reveals this map to be invariant in $v$ so that the matrix $\tau_{v}^{r} \tau_{v}$ has entries that are degree-18 $\mathcal{G}_{v}$ invariants. Hence, the matrix product has the $K$-expression

$$
\tau_{v}^{r} \tau_{v}=\Phi_{2}(v)^{9} T_{K} \quad \text { or } \quad\left(\tau_{v}^{r} \tau_{v}\right)^{-1}=\frac{T_{K}^{-1}}{\Phi_{2}(v)^{9}} .
$$

(See [Crass 2000] for the explicit form.) Also, note that

$$
\operatorname{det} T_{K}=\frac{\operatorname{det}\left(\tau_{v}^{r} \tau_{v}\right)}{\Phi_{2}(v)^{9 \cdot 7}}=\frac{\left(\operatorname{det} \tau_{v}\right)^{2}}{\Phi_{2}(v)^{63}}=t_{K} .
$$


Making use of (4.7) to express the transformation of basic equivariants yields

$$
\begin{aligned}
\phi_{1}(u) & =-4 \Phi_{2}(v)^{9} \tau_{v} \frac{T_{K}^{-1}}{\Phi_{2}(v)^{9}} \nabla_{w}^{r} \Phi_{2_{K}}(w) \\
& =-4 \tau_{v} T_{K}^{-1} \nabla_{w}^{r} \Phi_{2_{K}}(w) .
\end{aligned}
$$

As for the other relevant maps, similar calculations give

$$
\begin{aligned}
\phi_{2}(u) & =-\frac{8}{3} \Phi_{2}(v)^{12} \Phi_{3}(v) \tau_{v} \frac{T_{K}^{-1}}{\Phi_{2}(v)^{9}} \nabla_{w}^{r} \Phi_{3_{K}}(w) \\
& =-\frac{8}{3} \Phi_{2}(v)^{3} \Phi_{3}(v) \tau_{v} T_{K}^{-1} \nabla_{w}^{r} \Phi_{3_{K}}(w) \\
\phi_{4}(u) & =-\frac{8}{4} \Phi_{2}(v)^{21} \Phi_{3}(v) \tau_{v} \frac{T_{K}^{-1}}{\Phi_{2}(v)^{9}} \nabla_{w}^{r} \Phi_{5_{K}}(w) \\
& =-2 \Phi_{2}(v)^{12} \Phi_{3}(v) \tau_{v} T_{K}^{-1} \nabla_{w}^{r} \Phi_{5_{K}}(w) .
\end{aligned}
$$

Finally, we can identify a $K$-parametrized 4-map $g_{K}(w)$ that is conjugate to $g_{4}(u)$. The map's expression in basic terms appears after substitution into the formula found in Section 3.4 :

$$
\begin{aligned}
g_{4}(u)= & 84 \phi_{4}(u)-9 \Phi_{2}(u) \phi_{2}(u)-2 \Phi_{3}(u) \phi_{1}(u) \\
= & -8 \Phi_{2}^{12}(v) \Phi_{3}(v) \tau_{v} T_{K}^{-1}\left(21 \nabla_{w}^{r} \Phi_{5_{K}}(w)\right. \\
& \left.-3 \Phi_{2_{K}}(w) \nabla_{w}^{r} \Phi_{3_{K}}(w)-\Phi_{3_{K}}(w) \nabla_{w}^{r} \Phi_{2_{K}}(w)\right) .
\end{aligned}
$$

Thus, we have a $K$-parametrized family of 4-maps on $\mathbf{C P}_{w}^{6}$ :

$$
g_{K}(w)=T_{K}^{-1}\left(21 \nabla_{w}^{r} \Phi_{5_{K}}(w)-3 \Phi_{2_{K}}(w) \nabla_{w}^{r} \Phi_{3_{K}}(w)-\Phi_{3_{K}}(w) \nabla_{w}^{r} \Phi_{2_{K}}(w)\right)
$$

whose relation to the reference 4-map is

$$
g_{4}(u)=-8 \Phi_{2}^{12}(v) \Phi_{3}(v) \tau_{v} g_{K}(w)
$$

4.7. Root selection. Being conjugate to $g_{4}(u)$ each $g_{K}(w)$ shares the former's conjectured reliable dynamics. Accordingly, the attractor for each choice of $K$ is the 8-point orbit in the corresponding $\mathbf{C P}_{w}^{6}$ so that for almost every $w_{0} \in \mathbf{C P}_{w}^{6}$,

$$
g_{K}^{n}\left(w_{0}\right) \longrightarrow \tau_{v}^{-1} p_{\ell}^{8} \quad \text { for some 8-point } p_{\ell}^{8} \in \mathbf{C P}_{u}^{6} \text {. }
$$

To solve the resolvent $R_{K}$, the output of the iteration must link with the roots of $R_{K}$. From here, we see that solving $R_{K}$ amounts to inverting $\tau_{v}$ - the form problem in another guise. This is effectively what the dynamics of $g_{K}$ accomplishes with the assistance of a $\mathcal{G}_{8 \text { ! }}$ tool that I now describe.

The quadratic $\mathcal{S}_{7}$-invariants

$$
X_{k}^{2}(x)=-7 x_{k}^{2}+\sum_{i \neq k} x_{i}^{2}
$$

form a $\mathcal{G}_{8 \text { ! }}$ orbit of size eight. Recall that

$$
L_{k}(u)=X_{k}\left(\overline{H^{T}} u\right)
$$

and let

$$
Q_{k}(u)=X_{k}^{2}\left(\overline{H^{T}} u\right)
$$


be the $\mathcal{H}_{u}$ expression for $X_{k}^{2}$. Furthermore, each of the eight forms

$$
G_{k}(u)=Q_{k}(u)-\frac{3}{4} L_{k}(u)^{2} \quad k=1, \ldots, 8
$$

vanish at the 8 -points $p_{\ell}^{8}$ with $\ell \neq k$ but not at $p_{k}^{8}$.

Now, consider the rational function

$$
J_{v}(w)=\alpha \sum_{k=1}^{8} \frac{G_{k}\left(\tau_{v} w\right)}{\Phi_{2}\left(\tau_{v} w\right)} \frac{\Phi_{2}(v) L_{k}(v)}{\Phi_{3}(v)}=\alpha \sum_{k=1}^{8} \frac{G_{k}\left(\tau_{v} w\right)}{\Phi_{2}\left(\tau_{v} w\right)} \sigma_{k}(v)
$$

where $\alpha$ is a constant to be determined. Since the $v$-degree of the numerator and denominator is $21=2 \cdot 9+3$ while the $w$-degree is 2 , the function is rationally degree zero in both variables. At an 8-point $\tau_{v}^{-1} p_{\ell}^{8}$ in $\mathbf{C P}_{w}^{6}$ seven of the eight terms in $J_{v}$ vanish; this leaves

$$
\alpha \frac{G_{\ell}\left(p_{\ell}^{8}\right)}{\Phi_{2}\left(p_{\ell}^{8}\right)} \sigma_{\ell}(v)
$$

Choosing

$$
\alpha=\frac{\Phi_{2}\left(p_{k}^{8}\right)}{G_{k}\left(p_{k}^{8}\right)}=\frac{1}{48} \quad(k=1, \ldots, 8)
$$

"selects" the root $\sigma_{\ell}(v)$ of $R_{K}(s)$. Since the iterative "output" of $g_{K}(w)$ is a single 8-point in $\mathbf{C P}_{w}^{6}$, the dynamics produces one root.

To obtain a usable form of the root-selector $J_{v}(w)$, let

$$
\Gamma_{v}(w)=\sum_{k=1}^{8} G_{k}\left(\tau_{v} w\right) L_{k}(v) .
$$

Since $\mathcal{G}_{v}$ permutes its terms, $\Gamma_{v}$ is invariant under the action and hence, expressible in $K$ :

$$
\Gamma_{v}(w)=\Phi_{2}(v)^{8} \Phi_{3}(v) \Gamma_{K}(w) .
$$

(The explicit form of $\Gamma_{K}$ appears at Crass 2000.) Application of (4.5) yields

$$
\begin{aligned}
J_{v}(w) & =\frac{\Phi_{2}(v) \Gamma_{v}(w)}{48 \Phi_{3}(v) \Phi_{2}\left(\tau_{v} w\right)} \\
J_{K}(w) & =\frac{\Gamma_{K}(w)}{48 \Phi_{2_{K}}(w)} .
\end{aligned}
$$

4.8. The procedure summarized. At Crass 2000], there are Mathematica data files and a notebook that implement the iterative solution to the octic.

(1) Select a general 8-parameter octic $p(x)$.

(2) Tschirnhaus transform $p(x)$ into a member $R_{K}(s)$ of the 6-parameter family of $\mathcal{G}_{8 \text { ! }}$ octics - this determines values for $K_{1}, \ldots, K_{6}$ as well as the auxiliary parameter $\lambda$.

(3) For the selected $K$ values, compute the matrix $T_{K}$, the invariants $\Phi_{2_{K}}(w)$ and $\Phi_{3_{K}}(w)$, the 4-map $g_{K}(w)$, the form $\Gamma_{K}(w)$, and the root-selector $J_{K}(w)$.

(4) From an arbitrary initial point $w_{0}$ iterate $g_{K}$ until convergence:

$$
g_{K}^{n}\left(w_{0}\right) \longrightarrow w_{\infty}
$$

Conjecturally, the output $w_{\infty}$ is an 8-point in $\mathbf{C P}_{w}^{6}$.

(5) Compute a root $\sigma=J_{K}\left(w_{\infty}\right)$ of $R_{K}$.

(6) Transform $\sigma$ into a root of $p(x)$. 


\section{BEYOND THE OCTIC}

5.1. The general case. In the following discussion the $\mathcal{S}_{n}$ actions under consideration derive from permutation of coordinates on the $\mathcal{S}_{n}$-invariant hyperplanes

$$
\mathcal{H}^{n-1}=\left\{\sum_{k=1}^{n} x_{k}=0\right\} .
$$

These irreducible representations of $\mathcal{S}_{n}$ project to actions $\mathcal{G}_{n}$ ! on $\mathbf{P} \mathcal{H}^{n-1} \simeq \mathbf{C P}^{n-2}$.

For equations of degree $n \neq 8$, does the analogue of the octic-solving algorithm exist? Evidently, the reduction of the $n$th degree polynomial to an $(n-2)$-parameter family of $\mathcal{G}_{n}$ ! resolvents is general.

Query 5.1. Is there an $\mathcal{S}_{n}$-equivariant 4-map on $\mathbf{P} \mathcal{H}^{n-1}$ that, on a $\left(\begin{array}{l}n \\ 2\end{array}\right)$-line $\mathcal{L}^{1}$ (where all but two coordinates are equal):

- superattracts in the off-line directions

- restricts to a reliable map whose attractor consists of the pair of $n$-points on $\mathcal{L}^{1}$ ?

Query 5.2. If so, is the map expressible as

$$
z \longrightarrow z^{3} \frac{a z+b}{b z+c} \quad a, b \in \mathbf{R} \quad \text { and }|b|<|a|
$$

when restricted to $\mathcal{L}^{1}$ ?

The affine space $\left\{x_{n} \neq 0\right\}$ parametrized by

$$
\left[x_{1}, \ldots, x_{n-2},-\sum_{k=1}^{n-2} x_{k}-1,1\right]
$$

is tangent to $\mathbf{P} \mathcal{H}^{n-1}$ at the affine part of $\mathcal{L}^{1}$ given by

$$
[\zeta, \ldots, \zeta,(2-n) \zeta-1,1] \text {. }
$$

We can identify these spaces respectively with

$$
\left\{\left(x_{1}, \ldots, x_{n-2}\right)\right\} \simeq \mathbf{C}^{n-2} \text { and }\{(\zeta, \ldots, \zeta)\} \simeq \mathbf{C} .
$$

Definition 5.1. Abusing notation, let $\mathcal{L}^{1}$ be the affine part of an $\left(\begin{array}{l}n \\ 2\end{array}\right)$-line. Use $\mathcal{T}_{\mathcal{L}^{1}}$ to denote the tangent space to $\mathbf{P} \mathcal{H}^{n-1}$ along $\mathcal{L}^{1}$. Also,

$$
\mathcal{L}_{\perp}^{1}=\left\{\sum_{k=1}^{n-2} x_{k}=0\right\} \simeq \mathbf{C}^{n-3}
$$

is the (euclidean) orthogonal complement in $\mathcal{T}_{\mathcal{L}^{1}}$ to $\mathcal{L}^{1}$.

The subgroup $S_{\mathcal{L}^{1}}$ of $\mathcal{G}_{n \text { ! }}$ that stabilizes $\mathcal{T}_{\mathcal{L}^{1}}$ is isomorphic to $\mathcal{S}_{n-2}$ and acts by permutations on $\mathbf{C}^{n-2}$. The action of $S_{\mathcal{L}^{1}}$ fixes $\mathcal{T}_{\mathcal{L}^{1}}$ and $\mathcal{L}_{\perp}^{1}$ set-wise and fixes $\mathcal{L}^{1}$ in a point-wise manner.

Remark. In the treatment of $\mathcal{L}^{1}$ below, we need not worry about the point at infinity

$$
[1, \ldots, 1,2-n, 0],
$$

since this point is in the same $\mathcal{G}_{n !}$-orbit as the affine point

$$
[1, \ldots, 1,0,2-n]
$$


which we have identified with

$$
\frac{1}{2-n}(1, \ldots, 1)
$$

For $n \geq 5$, the family of 4-maps on $\mathbf{P} \mathcal{H}^{n-1}$ is, in homogeneous parameters,

$$
g_{\alpha}=\alpha_{1} f_{4}+\alpha_{2} F_{2} f_{2}+\alpha_{3} F_{3} f_{1}
$$

where definitions of $F_{k}$ and $f_{k}$ are obvious extensions from the $\mathcal{G}_{8 !}$ case. (For $n<5$, the family of 4-maps is not 3 -dimensional.) Do three parameters suffice to obtain a map $g_{\alpha}$ with jacobian matrix $g_{\alpha}^{\prime}(z)$ at $z$ such that

$$
g_{\alpha}^{\prime}(z) \mathcal{L}_{\perp}^{1}=0 \quad \text { for all } z \in \mathcal{L}^{1} ?
$$

First of all, symmetry demands that, for each $g_{\alpha}$ and all $z \in \mathcal{L}^{1}$, there is only one "off-line" eigenvalue of $g_{\alpha}^{\prime}(z)$.

Lemma 5.1. For an action $\mathcal{G}$ on $\mathbf{C P}^{n}$ and a $\mathcal{G}$-equivariant $f$ that is holomorphic at $a$, the jacobian $f^{\prime}(a)$ is equivariant on the tangent space $\mathcal{T}_{a} \simeq \mathbf{C}^{n}$ under the stabilizer $\mathcal{S}_{a}$ of a.

Proof. After treating several technical matters, the proof amounts to a simple calculation.

(1) Take $a$ to be $[0, \ldots, 0,1]$ and $\mathcal{T}_{a}$ to be $\left\{x_{n+1} \neq 0\right\}$ and lift them to

$$
\hat{a}=(0, \ldots, 0,1) \quad \text { and } \quad \widehat{\mathcal{T} a}=\left\{x_{n+1}=1\right\} .
$$

(2) Strictly speaking, $\mathcal{S}_{a}$ is a group of projective transformations. Here, we choose linear representatives $T$ of $\mathcal{S}_{a}$ that, as maps on $\mathbf{C}^{n+1}$, satisfy $T a=a$. This group of linear transformations acts on $\widehat{\mathcal{T}_{a}}$.

(3) For a homogeneous polynomial $G(x)$ of degree $r$,

$$
r G(x)=\left(\nabla_{x} G(x)\right)^{T} x
$$

is a familiar identity. Generalized to a rational map $g(x)$, the result is

$$
r g(x)=g^{\prime}(x) x .
$$

Given $T \in \mathcal{G}$, let $\hat{T}$ be a lift to $\mathbf{C}^{n+1}$. Then for all $x \in \mathbf{C}^{n+1}$,

$$
\begin{aligned}
\left(f^{\prime}(T x) T\right) x & =\left(f^{\prime}(T x)\right) T x \\
& =r f(T x) \\
& =T(r f(x)) \\
& =T\left(f^{\prime}(x) x\right) \\
& =\left(T f^{\prime}(x)\right) x .
\end{aligned}
$$

Thus,

$$
f^{\prime}(T x) T=T f^{\prime}(x) .
$$

In particular, when $x=a$ and $T \in \mathcal{S}_{a}$,

$$
f^{\prime}(a) T=f^{\prime}(T a) T=T f^{\prime}(a) .
$$

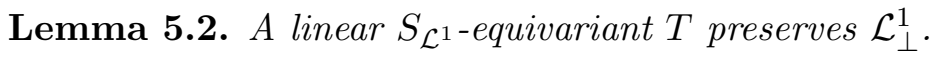


Proof. The transformation

$$
A: \mathcal{T}_{\mathcal{L}^{1}} \longrightarrow \mathcal{T}_{\mathcal{L}^{1}}
$$

that cyclically permutes coordinates according to $(12 \ldots(n-3)(n-2))$ has $n-2$ eigenspaces, namely, the lines $\mathcal{L}_{k}^{1}$ given by

$$
\mathcal{L}_{k}^{1}=\left\{t w_{k} \mid t \in \mathbf{C}, w_{k}=\left(\begin{array}{c}
1 \\
\omega^{k} \\
\omega^{2 k} \\
\vdots \\
\omega^{(n-3) k}
\end{array}\right), \omega=e^{2 \pi i /(n-2)}\right\} k=1, \ldots, n-2 .
$$

Note that $\mathcal{L}^{1}=\mathcal{L}_{n-2}^{1}$ and $\left\{w_{k} \mid k=1, \ldots, n-3\right\}$ is a basis for $\mathcal{L}_{\perp}^{1}$. Moreover, each line $\mathcal{L}_{k}^{1}$ has the eigenvalue $\omega^{(n-3) k}$.

Since $A \in S_{\mathcal{L}^{1}}$ and

$$
A\left(T w_{k}\right)=T\left(A w_{k}\right)=\omega^{(n-3) k} T w_{k},
$$

$T w_{k}$ is also an $\omega^{(n-3) k}$-eigenvector of $A$. Thus, each $\mathcal{L}_{k}^{1}$ is an eigenspace of $T$ so that $\mathcal{L}_{\perp}^{1}$ is $T$-invariant.

Proposition 5.1. For all $z \in \mathcal{L}^{1}$, the eigenvectors of the jacobian $g_{\alpha}^{\prime}(z)$ span $\mathcal{L}_{\perp}^{1}$. Moreover, all associated eigenvalues are equal, making $\mathcal{L}_{\perp}^{1}$ an eigenspace of $g_{\alpha}^{\prime}(z)$.

Proof. Recall that the stabilizer $\mathcal{S}_{\mathcal{L}^{1}}$ is isomorphic to $\mathcal{S}_{n-2}$. (The $n-2$ things that $\mathcal{S}_{\mathcal{L}^{1}}$ permutes are the vectors $w_{k}$ defined above.) By Lemmas 5.1 and 5.2. let $v \in \mathcal{L}_{\perp}^{1}$ be an eigenvector of $g_{\alpha}^{\prime}(z)$ with eigenvalue $\lambda$. For $A \in S_{\mathcal{L}^{1}}$, Lemma 5.1 gives

$$
g_{\alpha}^{\prime}(z) A v=A g_{\alpha}^{\prime}(z) v=\lambda A v
$$

Hence, $A v$ is also a $\lambda$-eigenvector. Clearly, $\left\{A v \mid A \in S_{\mathcal{L}^{1}}\right\}$ spans $\mathcal{L}_{\perp}^{1}$.

The question now is whether there is always some parameter-choice for which the eigenvalue of $\mathcal{L}_{\perp}^{1}$ vanishes for all $z$.

Proposition 5.2. For $n \geq 5$, there is a 4-map $g$ whose critical set includes the $\left(\begin{array}{l}n \\ 2\end{array}\right)$-lines. Moreover, at each point on an $\left(\begin{array}{l}n \\ 2\end{array}\right)$-line, $g$ is critical in every direction away from the line.

Proof. To facilitate exposition, we work in the linear space $\mathcal{H}^{n-1}$. For an arbitrary member of the family $g_{\alpha}$, select a lift $\widehat{g_{\alpha}}$ to $\mathcal{H}^{n-1}$. The line $\mathcal{L}^{1}$ lifts to the plane $\widehat{\mathcal{L}^{1}}$ parametrized by

$$
(x+y, \ldots, x+y,(1-n) x+y, x+(1-n) y) .
$$

Furthermore, the orthogonal complement $\widehat{\mathcal{L}^{1}}{ }_{\perp}$ in $\mathcal{H}^{n-1}$ is

$$
\left\{\sum_{k=1}^{n-2} x_{k}=0, x_{n-1}=x_{n}=0\right\} \text {. }
$$

By symmetry, we can consider a single line. Using the parametrization above, the pair of $n$-points on $\mathcal{L}^{1}$ correspond to the lines $x=0$ and $y=0$. Meanwhile, the line specified by

$$
x+y=0
$$

determines an element in one of the special orbits of $\left(\begin{array}{l}n \\ 2\end{array}\right)$-points. 
Proposition 5.1 implies that the characteristic polynomial for the jacobian of $\widehat{g_{\alpha}}$, when restricted to $\widehat{\mathcal{L}^{1}}$, has the form

$$
\left.\chi_{\widehat{g_{\alpha}}}{ }^{\prime}\right|_{\widehat{\mathcal{L}^{1}}}=\left.\operatorname{det}\left(t I_{n}-{\widehat{g_{\alpha}}}^{\prime}\right)\right|_{\widehat{\mathcal{L}^{1}}}=(t-A(x, y))^{n-3}\left(t^{2}-B(x, y) t+C(x, y)\right)
$$

Note that the factor

$$
t^{2}+B t+C
$$

is the characteristic polynomial of the jacobian of the map $\left.\widehat{g_{\alpha}}\right|_{\widehat{\mathcal{L}^{1}}}$. Hence,

$$
B(x, y)=B_{1} x^{3}+B_{2} x^{2} y+B_{3} x y^{2}+B_{4} y^{3}
$$

where the $B_{i}$ are linear in the parameters $\alpha_{j}$. It follows that

$$
A(x, y)=A_{1} x^{3}+A_{2} x^{2} y+A_{3} x y^{2}+A_{4} y^{3}
$$

where the $A_{i}$ are linear in the $\alpha_{j}$.

The polynomial $A$ gives the eigenvalue in the off-line directions in $\mathbf{P} \mathcal{H}^{n-1}$. The remaining factor corresponds to behavior along $\mathcal{L}^{1}$. Our interest here is $A$. In particular, we want to force it to vanish identically in $x$ and $y$.

Since $\chi_{\widehat{g_{\alpha}}}$ is invariant under permutation of coordinates, its restriction to $\widehat{\mathcal{L}^{1}}$ is invariant under the interchange $x \leftrightarrow y$ (which corresponds to the transposition $((n-1) n)$ in (5.8)). Accordingly, $A_{1}=A_{4}$ and $A_{2}=A_{3}$ so that

$$
\begin{aligned}
A & =A_{1}\left(x^{3}+y^{3}\right)+A_{2} x y(x+y) \\
& =(x+y)\left(A_{1}\left(x^{2}-x y+y^{2}\right)+A_{2} x y\right) .
\end{aligned}
$$

Thus, the $\left(\begin{array}{l}n \\ 2\end{array}\right)$-points are automatically critical away from $\mathcal{L}^{1}$.

We want to solve the linear equations $A_{1}=0$ and $A_{2}=0$ in the three parameters $\alpha_{1}, \alpha_{2}, \alpha_{3}$. Such a system has non-trivial solutions that give $A=0$ for all $x, y$.

\section{Remarks}

(1) The price of $A=0$ is at most two parameters. With the third parameter, we can only normalize the map. In the discussion that follows, we will discover that the cost is two parameters so that the resulting map is unique.

(2) Forcing $A_{1}=0$ is tantamount to making the pair of $n$-points on $\mathcal{L}^{1}$ critical. This "two-birds-with-one-stone" effect is what makes the procedure successful.

(3) Ostensibly, this argument is consistent with our obtaining a map that blows up at the $n$-points. At an $n$-point such a map would be critical in the "radial" direction in which $\mathcal{H}^{n-1}$ projects to $\mathbf{P} \mathcal{H}^{n-1}$. Such a circumstance would force $C=0$ when $x y=0$. Can the maps be critical in other directions as well? The preceding results and proofs provide for explicit calculation of the special 4-map. As a consequence, we see that the map does not blow up at the $n$-points. Furthermore, we derive the form of the map on an $\left(\begin{array}{l}n \\ 2\end{array}\right)$-line.

At first, we use the parametrization

$$
[x, \ldots, x, y,(2-n) x-y]
$$

for $\mathcal{L}^{1}$. Restricting the basic invariants to $\mathcal{L}^{1}$ gives

$$
\widetilde{F_{k}}=\left.F_{k}\right|_{\mathcal{L}^{1}}=(n-2) x^{k}+y^{k}+((2-n) x-y)^{k} .
$$


As for basic maps, note that

$$
f_{k}=\left[\left(f_{k}\right)_{1}, \ldots,\left(f_{k}\right)_{n}\right] \text { with }\left(f_{k}\right)_{\ell}=F_{k}-n x_{\ell}^{k} .
$$

Thus,

$$
\widetilde{f}_{k}=\left.f_{k}\right|_{\mathcal{L}^{1}}=\left[\widetilde{F_{k}}-n x^{k}, \ldots, \widetilde{F_{k}}-n x^{k}, \widetilde{F_{k}}-n y^{k}, \widetilde{F_{k}}-n((2-n) x-y)^{k}\right]
$$

and we can express the homogeneous map on $\mathcal{L}^{1}$ as

$$
\phi_{k}:[x, y] \longrightarrow\left[\widetilde{F_{k}}-n x^{k}, \widetilde{F_{k}}-n y^{k}\right] .
$$

Restricting the family of 4 -maps to $\mathcal{L}^{1}$ gives

$$
\widetilde{g_{\alpha}}=\alpha_{1} \widetilde{f}_{4}+\alpha_{2} \widetilde{F_{2}} \widetilde{f}_{2}+\alpha_{3} \widetilde{F_{3}} \widetilde{f}_{1}
$$

which, as a map on the line, is

$$
\begin{aligned}
\gamma_{\alpha}[x, y]= & {\left[\alpha_{1}\left(\widetilde{F_{4}}-n x^{4}\right)+\alpha_{2} \widetilde{F_{2}}\left(\widetilde{F_{2}}-n x^{2}\right)-n \alpha_{3} x \widetilde{F_{3}},\right.} \\
& \left.\alpha_{1}\left(\widetilde{F_{4}}-n y^{4}\right)+\alpha_{2} \widetilde{F_{2}}\left(\widetilde{F_{2}}-n y^{2}\right)-n \alpha_{3} y \widetilde{F_{3}}\right] .
\end{aligned}
$$

We can now determine three linear conditions on the $\alpha_{i}$ that correspond to

(1) normalizing the map so that the $n$-points are fixed in the affine sense - hence, they are not blown up

(2) making the map critical in every direction at the $n$-points

(3) making the map critical in every off-line direction along the $\left(\begin{array}{l}n \\ 2\end{array}\right)$-lines.

Consider the cases in turn.

(1) Since $[x, y]=[1,1]$ corresponds to an $n$-point, specify that

$$
1=\left(\gamma_{\alpha}[1,1]\right)_{1}=\left(n^{2}-2 n+2\right) \alpha_{1}+(n-1) n \alpha_{2}+(n-2) n\left((n-1) n \alpha_{3}\right) .
$$

(2) For the dehomogenized map

$$
f(z)=\frac{\left(\gamma_{\alpha}[z, 1]\right)_{1}}{\left(\gamma_{\alpha}[z, 1]\right)_{2}}
$$

set

$$
f^{\prime}(1)=0
$$

This requires

$$
-4 \alpha_{1}-2(n-1) n \alpha_{2}+(n-2)(n-1) n \alpha_{3}=0
$$

which amounts to $A_{1}=0$ in the proof of Proposition 5.2 .

(3) To arrive at $A_{2}=0$, evaluate the jacobian ${\widehat{g_{\alpha}}}^{\prime}(x)$ at a point $P$ on $\mathcal{L}^{1}$ other than an $n$-point or a $\left(\begin{array}{l}n \\ 2\end{array}\right)$-point - say, the point corresponding to $[x, y]=[1,0]$. Now, apply $g_{\alpha}^{\prime}(P)$ to the eigenvector

$$
v=(1,-1,0, \ldots, 0)
$$

in $\widehat{\mathcal{L}^{1}}{ }_{\perp}$. Since $v$ is an eigenvector, we can consider just the first component of $g_{\alpha}^{\prime}(P) v$. This component is

$$
g_{\alpha}^{\prime}(P)_{1,1}-g_{\alpha}^{\prime}(P)_{1,2}
$$


where

$$
\begin{aligned}
& g_{\alpha}^{\prime}(P)_{1,1}=-4(n-1) x^{3} \alpha_{1}-2 x\left(n x^{2}+(n-2) \widetilde{F_{2}}(1,0)\right) \alpha_{2}-n\left(3 x^{3}+\widetilde{F_{3}}(1,0)\right) \alpha_{3} \\
& g_{\alpha}^{\prime}(P)_{1,2}=4 x^{3} \alpha_{1}-2 x\left(n x^{2}-2 \widetilde{F_{2}}(1,0)\right) \alpha_{2}-3 n x^{3} \alpha_{3} .
\end{aligned}
$$

Thus, the condition for $A_{2}=0$ is

$$
n\left(-4 \alpha_{1}-2(n-2)(n-1) \alpha_{2}+(n-3)(n-2)(n-1) \alpha_{3}\right)=\left(g_{\alpha}^{\prime}(P) v\right)_{1}=0 .
$$

Solving (5.12), (5.13), and (5.14) yields a unique 4-map $g=g_{\alpha}$ with

$$
\begin{aligned}
\alpha_{1} & =\frac{1}{(n-4) n^{3}} \\
\alpha_{2} & =\frac{-6}{(n-4)(n-1) n^{4}} \\
\alpha_{3} & =\frac{-8}{(n-4)(n-2)(n-1) n^{4}} .
\end{aligned}
$$

Natural coordinates in which to express $g$ as a map on $\mathcal{L}^{1}$ are those of (5.8) where $x$ and $y$ are symmetrical. The result is

$$
\begin{aligned}
{[x, y] \longrightarrow } & {\left[\left(\gamma_{\alpha}[x+y,(1-n) x+y]\right)_{1}-\left(\gamma_{\alpha}[x+y,(1-n) x+y]\right)_{2},\right.} \\
& \left.(n-1)\left(\gamma_{\alpha}[x+y,(1-n) x+y]\right)_{1}-\left(\gamma_{\alpha}[x+y,(1-n) x+y]\right)_{2}\right] .
\end{aligned}
$$

Using the inhomogeneous coordinate $z=\frac{x}{y}$ the map becomes

$$
z \longrightarrow-\frac{(n-1) z-4}{4 z-(n-1)}
$$

in agreement with Section 3.4. As for dynamics the respective pair of $n$-points are 0 and $\infty$. Since $n-1 \geq 4$, the restricted map has 0 and $\infty$ as its only attractor. The respective basins are $\{|z|<1\}$ and $\{|z|>1\}$.

5.2. Another description. Using $n-1$ homogeneous coordinates, generalize to $\mathbf{C P}^{n-1}$ the $v$ coordinates that describe the $\mathcal{G}_{8 !}$-equivariant $g_{4}$. These place the $n$-points at

$$
p_{1}=[1,0, \ldots, 0], \ldots, p_{n-1}=[0, \ldots, 0,1], p_{n}=[1, \ldots, 1] .
$$

The coordinate change is given by

$$
x=P v \quad v=Q x
$$

where

$$
P=\left(p_{i j}\right)=\left\{\begin{array}{ll}
1-n & i=j \\
1 & i \neq j
\end{array} \quad \text { for } 1 \leq i \leq n, 1 \leq j \leq n-1\right.
$$

and $Q$ is the "inverse" of $P$ :

$$
Q=\left(q_{i j}\right)=\left\{\begin{array}{ll}
-1 & i=j \\
0 & i \neq j, j<n \\
1 & j=n
\end{array} \quad \text { for } 1 \leq i \leq n-1,1 \leq j \leq n .\right.
$$

Using $\left[v_{1}, \ldots, v_{n-1}\right]$ for this system, we define $S_{k}$ to be the $k$ th elementary symmetric function in $n-2$ variables and the coordinates

$$
\widehat{v_{k}}=\left(\ldots, v_{k-1}, v_{k+1}, \ldots\right)
$$


complementary to $v_{k}$. The stabilizer $\mathcal{G}_{(n-1)}$ ! of $p_{n}$ is the $\mathcal{S}_{n-1}$ group of permutations of the $v_{k}$. The order-2 transformation $Z_{n}$ that exchanges $p_{1}$ and $p_{n}$ while fixing the remaining $p_{k}$ generates $\mathcal{G}_{n \text { ! over }} \mathcal{G}_{(n-1) !}$. Note that $\left(\begin{array}{c}n-1 \\ 2\end{array}\right)$ of the $\left(\begin{array}{l}n \\ 2\end{array}\right)$-lines consist of points for which all but two coordinates vanish, while the remaining $n$ lines have points with all but one coordinate equal.

Let

$$
g(x)=\left[g_{1}(x), \ldots, g_{n}(x)\right]
$$

To compute the special 4-map

$$
\gamma(v)=Q(g(P v))=\left[g_{n}(P v)-g_{1}(P v), \ldots, g_{n}(P v)-g_{n-1}(P v)\right]
$$

in $v$ coordinates, we need to find only the first component of $\gamma$. Permutation symmetry in $v$ tends to the remaining components. Note that

$$
P v=\left(\begin{array}{c}
(1-n) v_{1}+S_{1}\left(\widehat{v_{1}}\right) \\
v_{1}+S_{1}\left(\widehat{v_{1}}\right)-n v_{2} \\
\vdots \\
v_{1}+S_{1}\left(\widehat{v_{1}}\right)-n v_{n-1} \\
v_{1}+S_{1}\left(\widehat{v_{1}}\right)
\end{array}\right)=\left(\begin{array}{c}
u_{1}-n v_{1} \\
u_{1}-n v_{2} \\
\vdots \\
u_{1}-n v_{n-1} \\
u_{1}
\end{array}\right)
$$

where $u_{1}=v_{1}+S_{1}\left(\widehat{v_{1}}\right)$. Application of (5.11) gives

$$
\begin{aligned}
\gamma_{1}(v)= & g_{n}(P v)-g_{1}(P v) \\
= & \left(\alpha_{1}\left(F_{4}(P v)-n u_{1}^{4}\right)+\alpha_{2}\left(F_{2}(P v)^{2}-n F_{2}(P v) u_{1}^{2}\right)+n \alpha_{3} F_{3}(P v) u_{1}\right) \\
& -\left(\alpha_{1}\left(F_{4}(P v)-n\left(u_{1}-n v_{1}\right)^{4}\right)+\alpha_{2}\left(F_{2}(P v)^{2}-n F_{2}(P v)\left(u_{1}-n v_{1}\right)^{2}\right)\right. \\
& \left.+n \alpha_{3} F_{3}(P v)\left(u_{1}-n v_{1}\right)\right) \\
= & n\left(\alpha_{1}\left(\left(u_{1}-n v_{1}\right)^{4}-u_{1}^{4}\right)+\alpha_{2} F_{2}(P v)\left(\left(u_{1}-n v_{1}\right)^{2}-u_{1}^{2}\right)\right. \\
& \left.+\alpha_{3} F_{3}(P v)\left(\left(u_{1}-n v_{1}\right)-u_{1}\right)\right) \\
= & -n^{2} v_{1}\left(\alpha_{1}\left(\left(u_{1}-n v_{1}\right)^{3}+\left(u_{1}-n v_{1}\right)^{2} u_{1}+\left(u_{1}-n v_{1}\right) u_{1}^{2}+u_{1}^{3}\right)\right. \\
& \left.+\alpha_{2} F_{2}(P v)\left(2 u_{1}-n v_{1}\right)+\alpha_{3} F_{3}(P v)\right) .
\end{aligned}
$$

Straightforward calculation yields

$$
\begin{aligned}
F_{2}(P v)= & u_{1}^{2}-n v_{1}^{2}-n S_{1}\left(\widehat{v_{1}}\right)^{2}+2 n S_{2}\left(\widehat{v_{1}}\right) \\
F_{3}(P v)= & 2 u_{1}^{3}-3 n u_{1} v_{1}^{2}+n^{2} v_{1}^{3}-3 n u_{1} S_{1}\left(\widehat{v_{1}}\right)^{2}+n^{2} S_{1}\left(\widehat{v_{1}}\right)^{3} \\
& +6 n u_{1} S_{2}\left(\widehat{v_{1}}\right)-3 n^{2} S_{1}\left(\widehat{v_{1}}\right) S_{2}\left(\widehat{v_{1}}\right)+3 n^{2} S_{3}\left(\widehat{v_{1}}\right) .
\end{aligned}
$$

Using (5.15), the substitution for $u_{1}$, and permutation in the $v_{i}$, the map takes the form

$$
\gamma(v)=\left[v_{1} T_{1}(v), \ldots, v_{n-1} T_{n-1}(v)\right]
$$

where

$$
T_{k}(v)=v_{k}^{3}-a_{2} v_{k}^{2} S_{1}\left(\widehat{v_{k}}\right)+a_{3} v_{k} S_{2}\left(\widehat{v_{k}}\right)-a_{4} S_{3}\left(\widehat{v_{k}}\right)
$$

and

$$
a_{2}=\frac{4}{n-1} \quad a_{3}=\frac{12}{(n-1)(n-2)} \quad a_{4}=\frac{24}{(n-1)(n-2)(n-4)}
$$




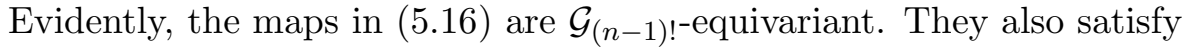

$$
Z_{n} \circ g=g \circ Z_{n} \quad \text { for all } n \geq 5 .
$$

5.3. Revisiting the quintic. In solving the quintic, Crass 2001 harnesses the dynamics of a degree- 6 map whose behavior is similar to the maps treated in the present paper. The $\mathcal{G}_{5 !}$ equivariant 4-map is also a good candidate for inclusion in a quintic-solving algorithm. Having lower degree, its global dynamics might be more tractable. For instance, this map has a kind of critical finiteness that other $\mathcal{G}_{n}$ ! 4 -maps do not share. I plan to examine this map in more dynamical detail in an upcoming paper.

\section{BASIN PORTRAITS}

The plots that follow are productions of the program Dynamics 2 running on a Dell Dimension XPS with a Pentium II processor. Its BA and BAS routines produced the pictures. (See the manual [Nusse and Yorke 1998.) Briefly, each procedure divides the screen into a grid of cells and then colors each cell according to which attracting point its trajectory approaches. If it finds no such attractor after a specified number of iterationsusually 60 , the cell is black. The BA algorithm looks for an attractor whereas BAS requires the user to specify a candidate attracting set of points. Each portrait exhibits the highest resolution available - a $720 \times 720$ grid.

All of the images show $g_{4}$ restricted to either a $\mathbf{C P}^{1}$ or $\mathbf{R P}^{2}$ that it preserves. Some restricted maps have attracting sites that are not the 8-points. However, none of the detected "restricted attractors" other than the 8-points themselves are overall attractors with 6-dimensional basins.

Figure 2, When restricted to the line $\mathcal{L}_{168}^{1}, g_{4}$ has only trivial symmetry. Consequently, there is no natural choice of coordinates in which to express the restriction to the line. With an 8 -point at 0 and a 28 -point $q_{i j}^{28}$ at $\infty$, the map takes the form

$$
z \longrightarrow-\frac{z^{3}(3 z+4)}{4\left(z^{2}+8 z+14\right)}
$$

The two basins that appear are associated with the 8-point and 28-point. The latter is an "equatorial" saddle point on the 28-line $\mathcal{L}_{28_{i j}}^{1}$. The basin in $\mathcal{L}_{168}^{1}$ is a slice of its 5dimensional stable manifold. The reflective symmetry that appears is due to the map's anti-holomorphic equivariance. (See Section 3.4.)

Not pictured is the portrait for $\mathcal{L}_{210}^{1}$ on which the map takes the form

$$
z \longrightarrow-z^{2} \frac{3 z+1}{z+3}
$$

As in the case of a 28-line, this map has two basins: $\{|z|<1\}$ and $\{|z|>1\}$. The attracting points are of the types

$$
[1,1,-1,-1,0,0,0,0] \quad \text { and } \quad[1,1,1,1,-1,-1,-1,-1] \text {. }
$$

Overall, these behave as saddles. In fact, at the latter point, $g_{4}$ blows up onto the associated hyperplane

$$
\left\{x_{1}+x_{2}+x_{3}+x_{4}=0\right\} \cap\left\{x_{5}+x_{6}+x_{7}+x_{8}=0\right\} .
$$


Figure 3. This shows $g_{4}$ on $\mathcal{L}_{280}^{1}$ in the form

$$
z \longrightarrow \frac{8 z^{3}}{5 z^{4}+6 z^{2}-3}
$$

The basin at 0 is due to a 28-point $p_{i j}^{28}$. This point belongs to $\mathcal{L}_{28_{i j}}^{1}$ and is repelling on that line. Thus, the basin pictured here is a 1-dimensional slice of the 5-dimensional stable manifold attached to the point. At \pm 1 we see petals due to rationally indifferent points of type

$$
[3,3,3,3,3,-5,-5,-5] \text {. }
$$

The indifferent local behavior at these points is evident in this and several subsequent images.

Figure 4, On $\mathcal{M}_{280}^{1}, g_{4}$ is a polynomial map expressible by

$$
z \longrightarrow-z\left(7 z^{2}-5 z+1\right) \text {. }
$$

Here, an 8 -point is at $\infty$ (the dark blue basin) and, once again, a rationally indifferent point of type

$$
[3,3,3,3,3,-5,-5,-5]
$$

appears at 0 .

Figure 5. We see the $\mathcal{S}_{3}$-symmetric restriction to the $\mathbf{R} \mathbf{P}^{2}$ intersection of $\mathcal{L}_{56}^{2}$ and $\mathcal{R}$. The three basins result from a triple of 8-points arranged symmetrically on the unit circle. A line of reflective symmetry passes through each of the 8-points and $(0,0)$-which corresponds to a point of type

$$
[3,3,3,3,3,-5,-5,-5]
$$

These lines are $\mathbf{R} \mathbf{P}^{1}$ intersections of an $\mathcal{L}_{168}^{1}$ with the $\mathbf{R} \mathbf{P}^{2}$. Each $\mathbf{R P}^{1}$ contains points in the basin of an 8-point and as well as points in the boundary between the basins of the other two 8-points. This interval lies in the basin of a 28-point $q_{i j}^{28}$ as seen in Figure 2 ,

Figure 6. The image displays the basins of the $\mathcal{S}_{4}$-symmetric map on the intersection of $\mathcal{L}_{105}^{2}$ and $\mathcal{R}$. Each $\mathcal{L}_{105}^{2}$ is canonically associated with a $\mathbf{C P}^{3}, \mathcal{L}_{105}^{3}$, whose points have coordinates that come in four mutually negative pairs:

$$
\begin{array}{ll}
\left\{x_{i}=x_{j}\right\} \cap\left\{x_{k}=x_{\ell}\right\} & \longleftrightarrow\left\{x_{i}=-x_{j}\right\} \cap\left\{x_{k}=-x_{\ell}\right\} \\
\cap\left\{x_{m}=x_{n}\right\} \cap\left\{x_{p}=x_{q}\right\} & \cap\left\{x_{m}=-x_{n}\right\} \cap\left\{x_{p}=-x_{q}\right\} .
\end{array}
$$

Under $g_{4}, \mathcal{L}_{105}^{3}$ blows down to $\mathcal{L}_{105}^{2}$. Furthermore, each point in the orbit of

$$
[1,-1,1,-1,1,-1,1,-1]
$$

belongs to 24 of the $\mathcal{L}_{105}^{3}$ so that the blowing-down of each $\mathbf{C P}^{3}$ forces all coordinates of the image to be equal. Hence, they must all vanish.

To describe things explicitly, take the plane

$$
\mathcal{L}_{105}^{2}=\left\{x_{1}=x_{2}\right\} \cap\left\{x_{3}=x_{4}\right\} \cap\left\{x_{5}=x_{6}\right\} \cap\left\{x_{7}=x_{8}\right\}
$$

parametrized by

$[x, y, z] \longrightarrow[x+y+z, x+y+z, x-y-z, x-y-z,-x-y+z,-x-y+z,-x+y-z,-x+y-z]$.

There is a 3 -point orbit at

$$
[1,0,0],[0,1,0],[0,0,1]
$$


corresponding to the points

$$
[1,1,1,1,-1,-1,-1,-1],[1,1,-1,-1,-1,-1,1,1],[1,1,-1,-1,1,1,-1,-1]
$$

and a 4 -point orbit at $[ \pm 1, \pm 1,1]$ corresponding to the 28 -points

$$
\begin{aligned}
& q_{12}^{28}=[-3,-3,1,1,1,1,1,1], q_{34}^{28}=[1,1,-3,-3,1,1,1,1] \\
& q_{56}^{28}=[1,1,1,1,-3,-3,1,1], q_{78}^{28}=[1,1,1,1,1,1,-3,-3] .
\end{aligned}
$$

Associated with the 3 -points are the lines

$$
\{x=0\},\{y=0\},\{z=0\}
$$

corresponding to intersections with three of the $\mathcal{L}_{105}^{3}$ :

$$
[u, u,-u,-u, v, v,-v,-v],[u, u, v, v,-v,-v,-u,-u], \quad[u, u, v, v,-u,-u,-v,-v] .
$$

In these coordinates, the map has the expression

$$
[x, y, z] \longrightarrow\left[y z\left(4 x^{2}+y^{2}+z^{2}\right), x z\left(x^{2}+4 y^{2}+z^{2}\right), x y\left(x^{2}+y^{2}+4 z^{2}\right)\right] .
$$

The attractor here seems to consist of four 28-points $q_{i j}^{28}$ at $( \pm 1, \pm 1)$. The points $q_{i j}^{28}$ are on $\mathcal{L}_{28_{i j}}^{1}$ and, thus, repel in directions away from the picture-plane. Here we see off-line directions relative to $\mathcal{L}_{28_{i j}}^{1}$. Each $q_{i j}^{28}$ lies on 15 of the $\mathcal{L}_{105}^{2}$ and their "restricted" basins in the $\mathbf{C P}^{2}$ belong to their stable manifolds as well as to the overall Julia set of $g_{4}$. Hence, these pieces of the Julia set have zero measure in $\mathbf{C P}^{6}$. As for real dynamics, the basins appear to be the four quadrants

$$
\{x>0, y>0\},\{x<0, y>0\},\{x<0, y<0\},\{x>0, y<0\}
$$

which are forward and, thus, totally invariant. Accordingly, the coordinate axes form the basin boundaries.

Finally, each coordinate axis blows down to its companion point while a 3-point blows up onto its associated axis.

Figure [7. For the $\mathbf{Z}_{2}^{2}$ map on the intersection of $\mathcal{L}_{280}^{2}$ and $\mathcal{R}$ the two prominent basins belong to a pair of 8-points at $( \pm 1,0)$. The other two attracted regions are associated with a pair of indifferent points (both eigenvalues are -1 ) of type

$$
[3,3,3,3,3,-5,-5,-5] \text {. }
$$

Figure 8, On the $\mathbf{Z}_{2}$-symmetric restriction to the intersection of $\mathcal{L}_{420}^{2}$ and $\mathcal{R}$, two 8-points at $( \pm 1,0)$ and a $q_{i j}^{28}$ at $(0,-1)$ account for the basins.

Figure 9, As for the behavior of the $\mathbf{Z}_{2}$-symmetric map on the intersection of $\mathcal{L}_{840}^{2}$ and $\mathcal{R}$, there is an 8-point at $(0,0)$ and a pair of 28-points $q_{i j}^{28}, q_{k \ell}^{28}$ at $( \pm 1,1)$. Trajectories starting in the thin vertical black strip near the bottom middle require too many iterations in order to converge to one of the three attractors for the BA routine to detect attraction. Experimental results suggest that this region is filled with rather small components of the three basins. 


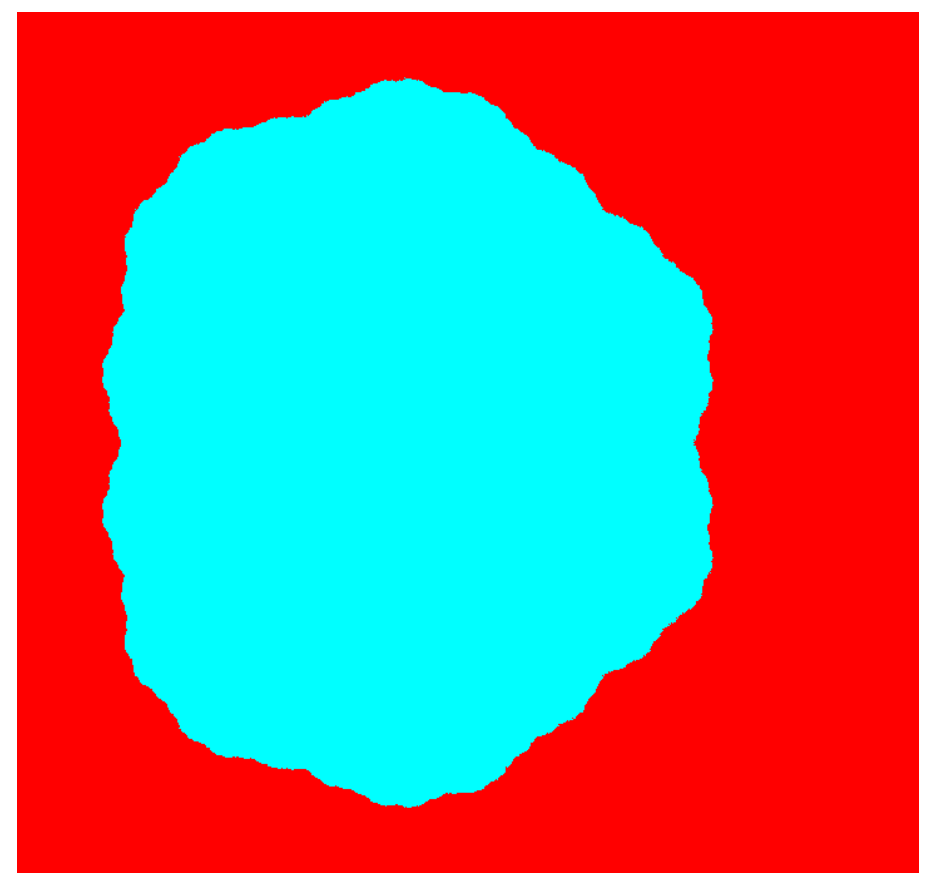

Figure 2. Dynamics of $g_{4}$ on $\mathcal{L}_{168}^{1}$

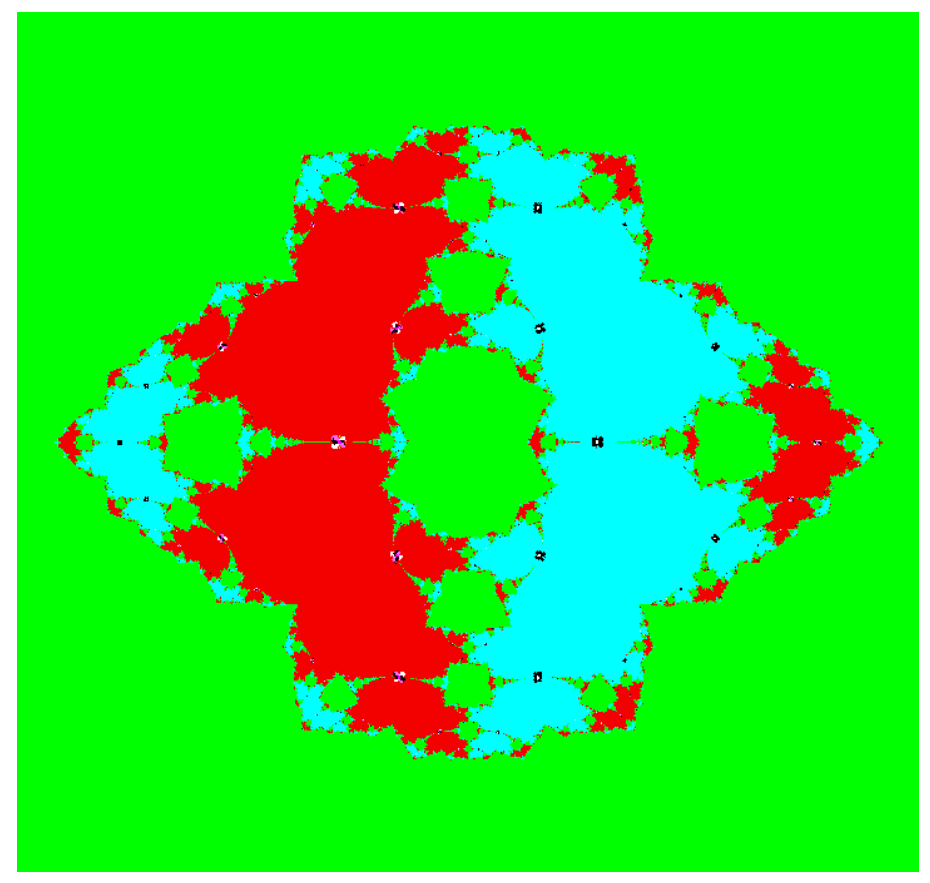

Figure 3. Dynamics of $g_{4}$ on $\mathcal{L}_{280}^{1}$ 


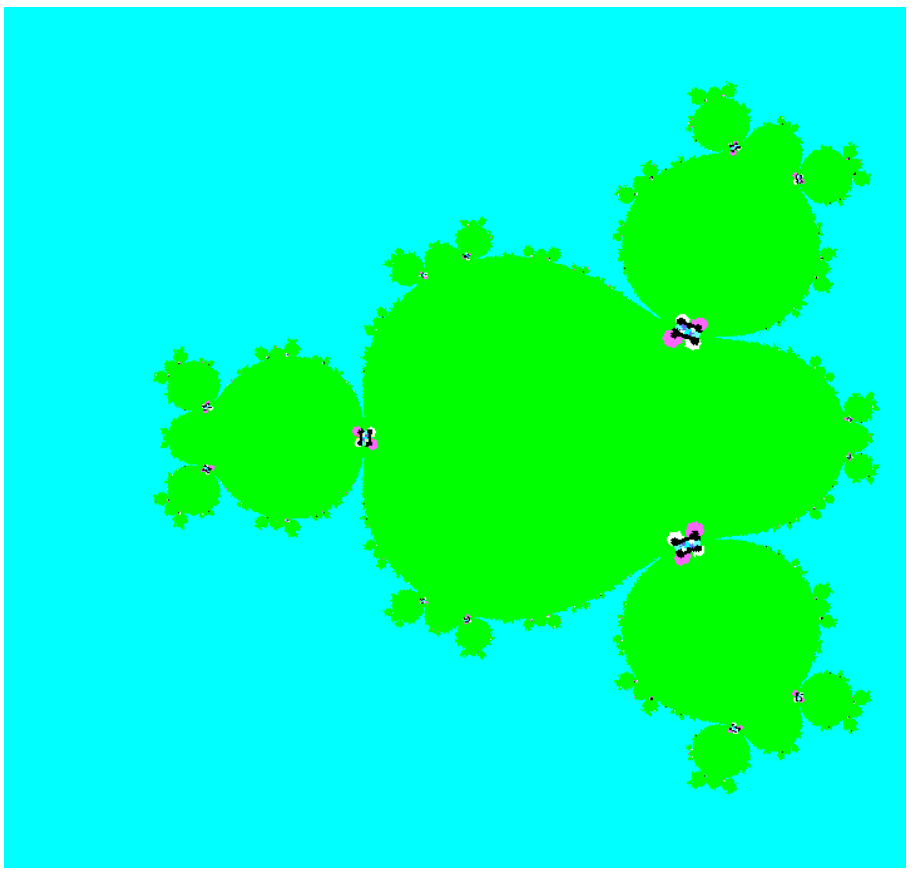

Figure 4. Dynamics of $g_{4}$ on $\mathcal{M}_{280}^{1}$

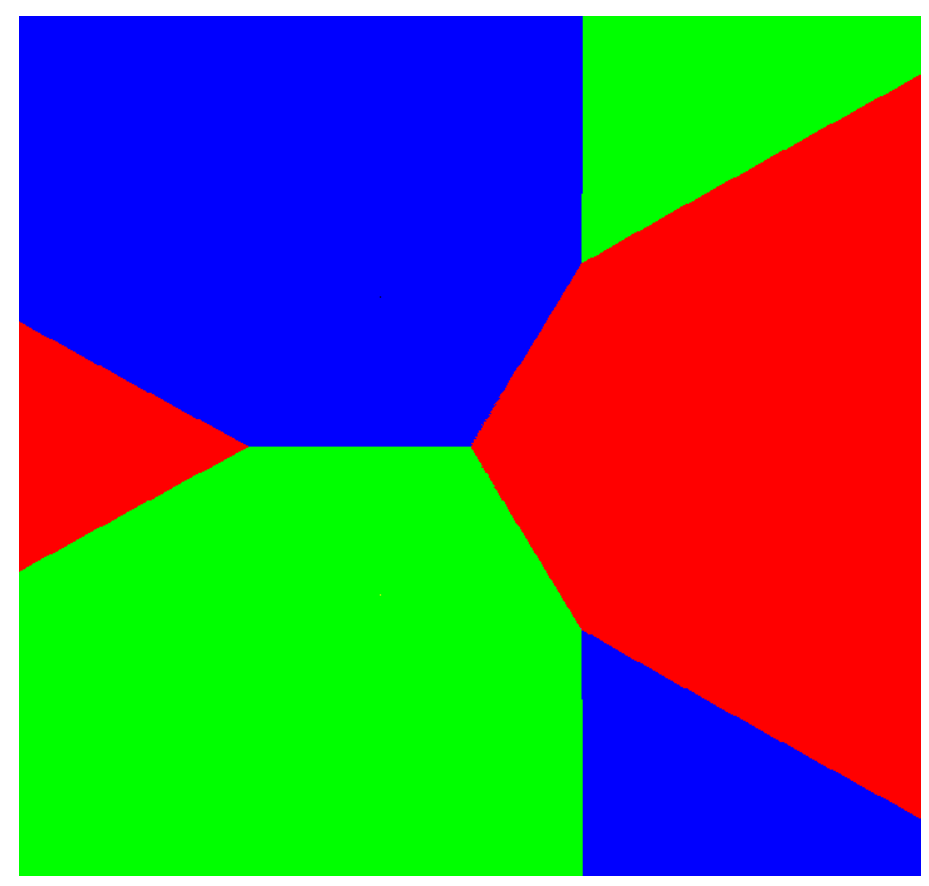

Figure 5. Dynamics of $g_{4}$ on $\mathcal{L}_{56}^{2} \cap \mathcal{R}$ 


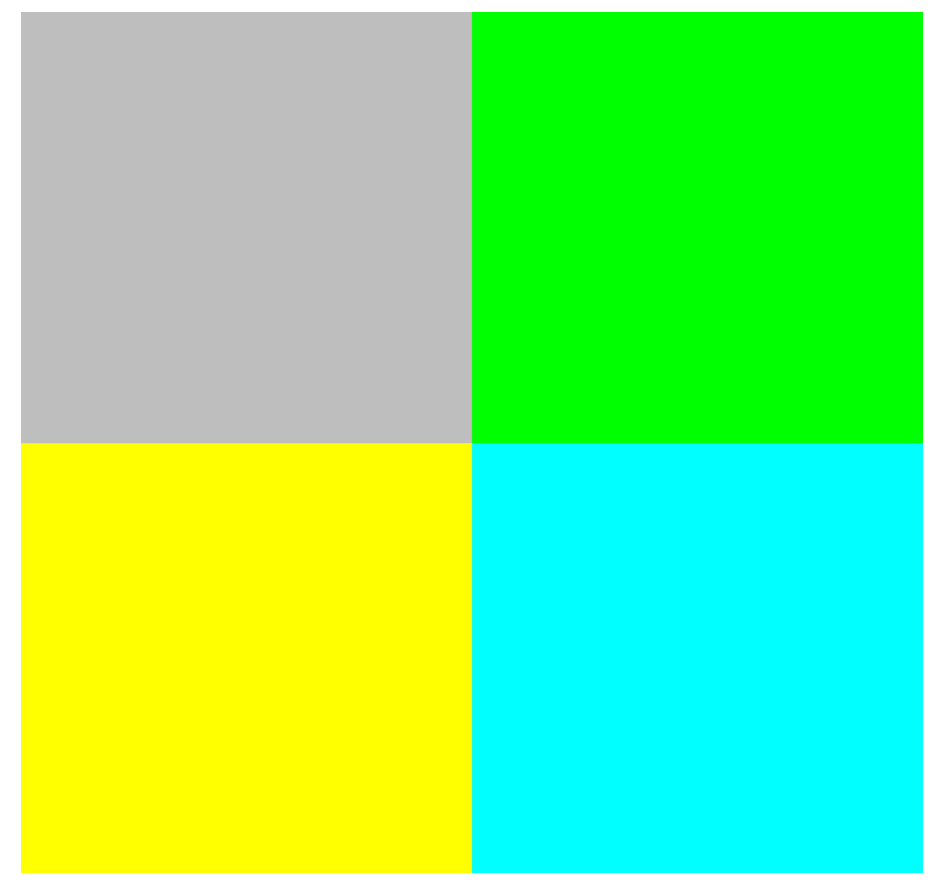

Figure 6. Dynamics of $g_{4}$ on $\mathcal{L}_{105}^{2} \cap \mathcal{R}$

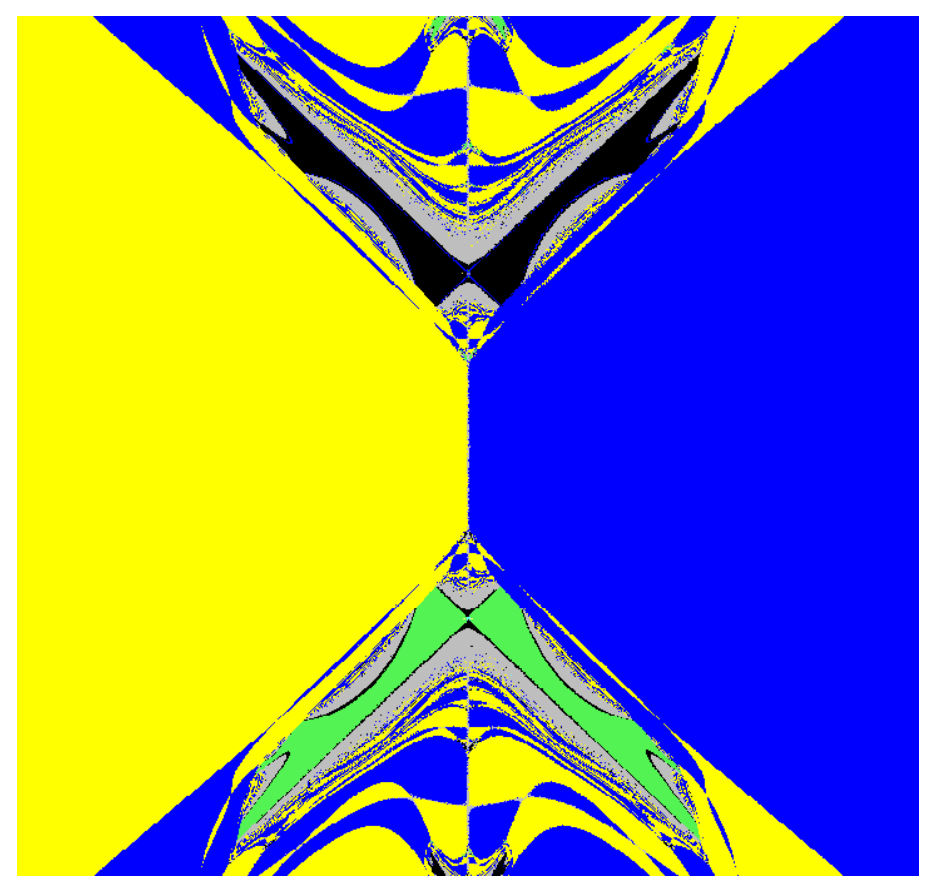

Figure 7. Dynamics of $g_{4}$ on $\mathcal{L}_{280}^{2} \cap \mathcal{R}$ 


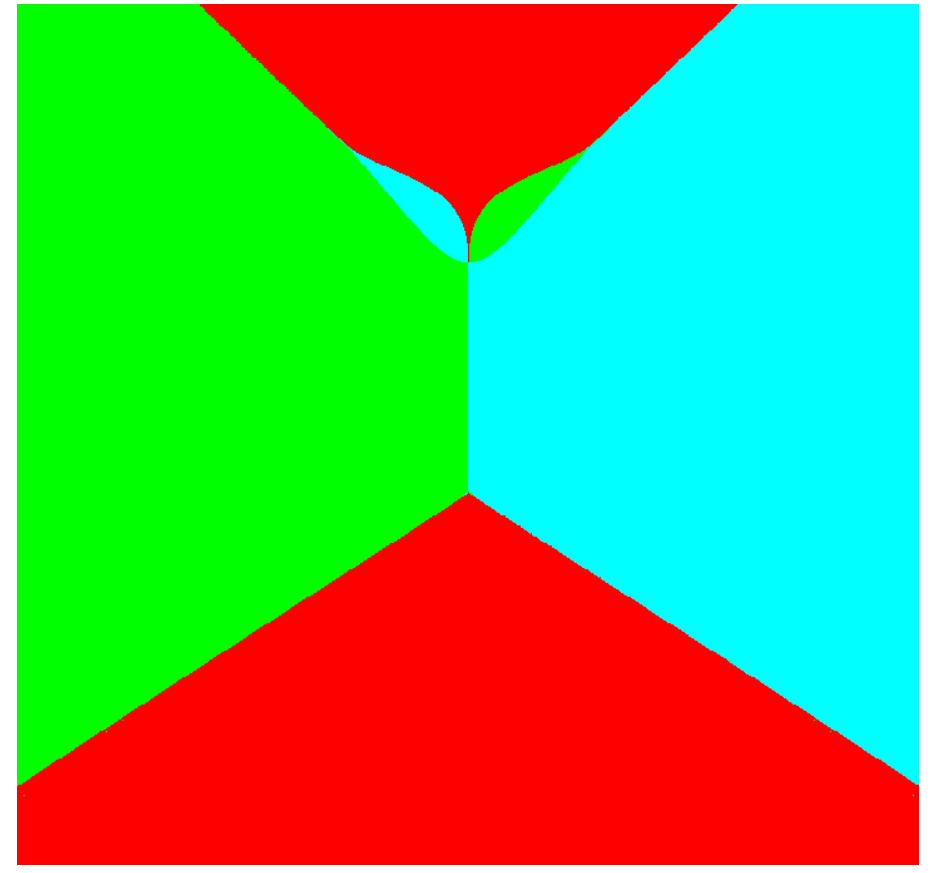

FiguRe 8. Dynamics of $g_{4}$ on $\mathcal{L}_{420}^{2} \cap \mathcal{R}$

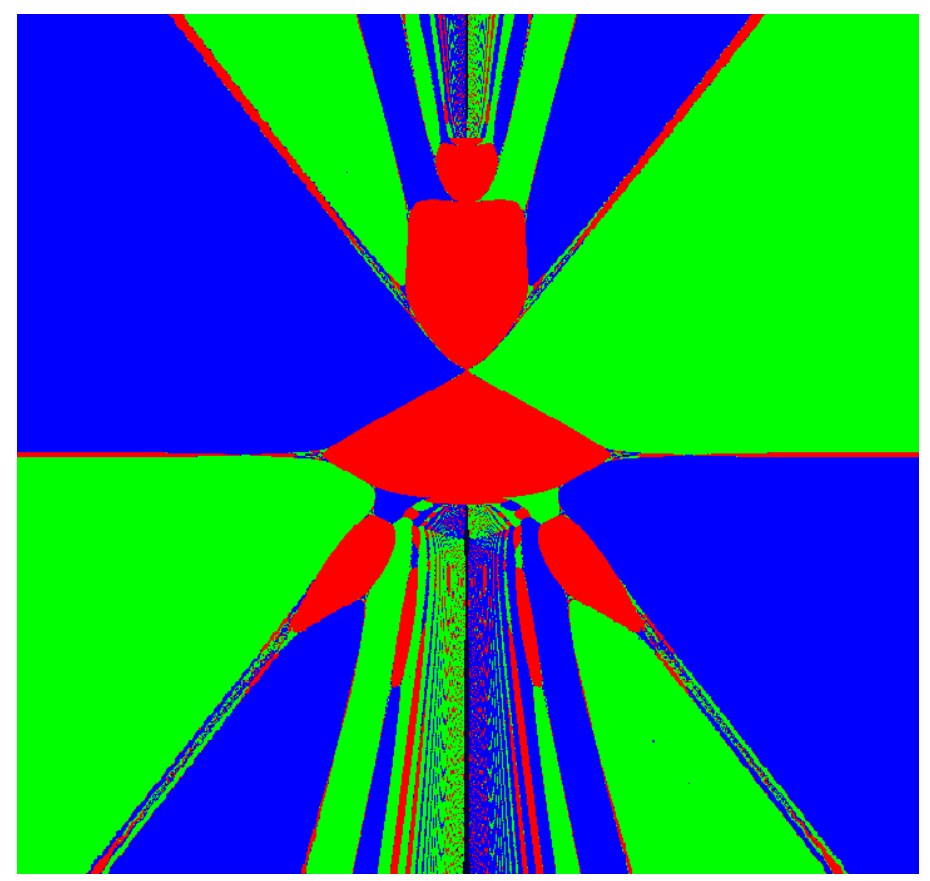

Figure 9. Dynamics of $g_{4}$ on $\mathcal{L}_{840}^{2} \cap \mathcal{R}$ 


\section{REFERENCES}

[Crass 1999] S. Crass. Solving the sextic by iteration: A study in complex geometry and dynamics. Experiment. Math. 8 (1999) No. 3, 209-240. Preprint at arxiv.org/abs/math.DS/9903111.

[Crass 2001] S. Crass. Solving the quintic by iteration in three dimensions. Experiment. Math. 10 (2001) No.1, 1-24.

Preprint at arxiv.org/abs/math.DS/9903054.

[Crass 2000] S. Crass. Mathematica notebooks and supporting files for the solution of the octic. See csulb.edu/ scrass/math/octic/Solve.

[Klein 1913] F. Klein. Lectures on the Icosahedron and the Solutions of Equations of the Fifth Degree, Kegan Paul, London, 1913. Reprint by Dover, New York. Translation by G. Morrice of Vorlesungen über das Ikosaeder und die Auflösungen der Gleichungen vom fünften Grade, Teubner, Leipzig, 1884.

[Nusse and Yorke 1998] H. Nusse and J. Yorke. Dynamics: Numerical Explorations, 2e Springer-Verlag, 1998. Computer program Dynamics 2 by B. Hunt and E. Kostelich.

[Orlik and Terao 1992] P. Orlik and H. Terao. Arrangements of Hyperplanes. Springer-Verlag, 1992.

[Shephard and Todd 1954] G. C. Shephard and T. A. Todd. Finite unitary reflection groups. Canad. J. Math. 6 (1954), 274-304.

[Wiman 1899] A. Wiman. Ueber die Darstellung der symmetrischen und alternirenden Vertauschungsgruppen als Collineationsgruppen von möglichst geringer Dimensionzahl. Mathematische Annalen 52 (1899), 243-270.

Mathematics Department, California State University, Long Beach, Long Beach, CA 90840, E-mail address: scrass@csulb.edu 